\title{
Correcting Smad1/5/8, mTOR, and VEGFR2 treats pathology in hereditary hemorrhagic telangiectasia models
}

\author{
Santiago Ruiz, ${ }^{1}$ Haitian Zhao, ${ }^{1}$ Pallavi Chandakkar, ${ }^{1}$ Julien Papoin, ${ }^{2}$ Hyunwoo Choi, ${ }^{3}$ Aya Nomura-Kitabayashi, ${ }^{1}$ Radhika Patel, ${ }^{1}$ \\ Matthew Gillen, ${ }^{1}$ Li Diao, ${ }^{4}$ Prodyot K. Chatterjee, ${ }^{5}$ Mingzhu He, ${ }^{6}$ Yousef Al-Abed, ${ }^{6,7}$ Ping Wang, ${ }^{4,7}$ Christine N. Metz, ${ }^{5,7}$ S. Paul Oh, ${ }^{3}$ \\ Lionel Blanc, ${ }^{2,7}$ Fabien Campagne, ${ }^{8,9}$ and Philippe Marambaud ${ }^{1,7}$
}

\begin{abstract}
'Litwin-Zucker Center for Alzheimer's Disease and Memory Disorders and 'Center for Autoimmune, Musculoskeletal and Hematopoietic Diseases, The Feinstein Institutes for Medical Research, Northwell Health, Manhasset, New York, USA. ${ }^{3}$ Barrow Aneurysm and AVM Research Center, Department of Neurobiology, Barrow Neurological Institute, Phoenix, Arizona, USA. ${ }^{4}$ Center for Immunology and Inflammation, ${ }^{5}$ Institute of Molecular Medicine, and ${ }^{6}$ Center for Molecular Innovation, The Feinstein Institutes for Medical Research, Manhasset, New York, USA. ${ }^{7}$ Donald and Barbara Zucker School of Medicine at Hofstra/Northwell, Hempstead, New York, USA. ${ }^{8}$ The HRH Prince Alwaleed Bin Talal Bin Abdulaziz Alsaud Institute for Computational Biomedicine and ${ }^{9}$ Department of Physiology and Biophysics, Weill Cornell Medical College, New York, New York, USA.
\end{abstract}

\begin{abstract}
Hereditary hemorrhagic telangiectasia (HHT), a genetic bleeding disorder leading to systemic arteriovenous malformations (AVMs), is caused by loss-of-function mutations in the ALK1/ENG/Smad1/5/8 pathway. Evidence suggests that HHT pathogenesis strongly relies on overactivated PI3K/Akt/mTOR and VEGFR2 pathways in endothelial cells (ECs). In the BMP9/10-immunoblocked (BMP9/10ib) neonatal mouse model of HHT, we report here that the mTOR inhibitor, sirolimus, and the receptor tyrosine kinase inhibitor, nintedanib, could synergistically fully block, but also reversed, retinal AVMs to avert retinal bleeding and anemia. Sirolimus plus nintedanib prevented vascular pathology in the oral mucosa, lungs, and liver of the BMP9/10ib mice, as well as significantly reduced gastrointestinal bleeding and anemia in inducible ALK1-deficient adult mice. Mechanistically, in vivo in BMP9/10ib mouse ECs, sirolimus and nintedanib blocked the overactivation of mTOR and VEGFR2, respectively. Furthermore, we found that sirolimus activated ALK2-mediated Smad1/5/8 signaling in primary ECs - including in HHT patient blood outgrowth ECs - and partially rescued Smad1/5/8 activity in vivo in BMPg/10ib mouse ECs. These data demonstrate that the combined correction of endothelial Smad1/5/8, mTOR, and VEGFR2 pathways opposes HHT pathogenesis. Repurposing of sirolimus plus nintedanib might provide therapeutic benefit in patients with HHT.
\end{abstract}

\section{Introduction}

Hereditary hemorrhagic telangiectasia (HHT), also known as Rendu-Osler-Weber syndrome, is an autosomal dominant genetic disease with a prevalence of approximately 1 in 5,000 individuals (1). HHT is a hemorrhagic vascular dysplasia characterized by the development of mucocutaneous telangiectasias and arteriovenous malformations (AVMs) in multiple tissues and organs. Particularly affected is the vasculature of the liver, lungs, and oronasal and gastrointestinal (GI) mucosa. In its most severe manifestations, HHT can lead to highly debilitating and life-threatening events, such as severe epistaxis and internal bleeding. HHT is also associated with secondary complications, which include anemia, cerebral abscess, and embolism following pulmonary AVMs, as well as high-output cardiac failure consecutive to liver AVMs $(2,3)$.

At least $85 \%$ of patients with HHT carry disease-causing lossof-function mutations in the genes ENG (encoding endoglin) or

Conflict of interest: The Feinstein Institutes for Medical Research has filed a US provisional patent application (no. 62/736,564) titled "Combined sirolimus and nintedanib therapy for vascular lesions and hereditary hemorrhagic telangiectasia." Copyright: (5) 2020, American Society for Clinical Investigation.

Submitted: January 15, 2019; Accepted: October 31, 2019; Published: January 21, 2020 Reference information: J Clin Invest. 2020;130(2):942-957.

https://doi.org/10.1172/JCI127425.
ACVRL1 (encoding activin receptor-like kinase 1, ALK1), which define the 2 disease subtypes: HHT1 (OMIM \#187300) and HHT2 (OMIM \#600376), respectively $(4,5)$. Mutations have also been reported in SMAD4 (6) (encoding Smad4) and GDF2 (7) (encoding bone morphogenetic protein 9, BMP9), which cause rare and atypical forms of the disease called juvenile polyposis/HHT combined syndrome (OMIM \#175050) and HHT-like vascular anomaly syndrome (OMIM \#615506), respectively. BMP9, ALK1, endoglin, and Smad 4 are members of the transforming growth factor $\beta$ signaling superfamily, and all functionally interact in the same signal transduction axis (8). The cell surface receptor complex composed of the coreceptor endoglin, the endothelial BMP type I receptor ALK1, and a BMP type II receptor (e.g., BMPR2) is activated by sequential binding to the circulating ligands BMP9 and BMP10 (9-11). Mutations in BMPR2 cause familial pulmonary arterial hypertension $(\mathrm{PAH})$, which can be observed in some patients with HHT2 (12), further supporting the notion that ALK1 and BMPR2 functionally interact. ALK1-endoglin receptor activation leads to phosphorylation of the signal transducers Smad1, Smad5, and Smad8 to trigger the formation of Smad1/5/8-Smad 4 complexes that translocate into the nucleus to control specific gene expression programs (13).

HHT pathogenesis is triggered by a reduction in $\mathrm{Smad1} / 5 / 8$ signaling in ECs. Indeed, HHT-causing mutations decrease 
Smad1/5/8 response to ALK1-endoglin receptor activation by BMP9 (14-16). Consistent with this model, ALK1, endoglin, or Smad4 inactivation in mice and zebrafish leads to vascular defects, which include hypervascularization and AVMs (17-25). Downstream from ALK1-endoglin receptor loss of function, the exact pathways involved in HHT pathogenesis and ultimately, AVM development - i.e., the formation of direct shunts between an artery and a vein - remain incompletely understood (26). However, concordant studies have shown that HHT is associated with abnormal reactivation of angiogenesis (27) and that overactivated proangiogenic pathways, such as VEGF signaling, are required for the development of the vascular pathology of HHT models $(28,29)$. In vitro data in cell cultures have further revealed that VEGFR2 phosphorylation and activity were increased upon ALK1 silencing (30), and that endoglin silencing affected VEGFR2 trafficking to increase its downstream signaling (31). Transcriptomic data in ECs have also demonstrated that ALK1 negatively controlled VEGFR2 (KDR gene) expression (32, 33). In vivo, Vegfr2 knockdown was reported to block hypervascularization and AVMs in Alk ${ }^{\mathrm{i} \triangle \mathrm{EC}}$ mice (30) and pharmacological inhibition of VEGFR2 reduced disease severity in $E n g^{\mathrm{i} \triangle \mathrm{EC}}$ mice (31). Also, recent clinical trials have indicated that intravenous treatment with the VEGF-blocking antibody, bevacizumab, provided some therapeutic benefits in patients severely affected by $\operatorname{HHT}(34,35)$. In summary, although the field has not reached a consensus on the exact mechanism by which ALK1-endoglin loss of function activates VEGF signaling, there is strong evidence that VEGF/VEGFR2 signaling is a key contributor to the pathogenic process of HHT.

$\mathrm{PI} 3 \mathrm{~K} /$ Akt overactivation is also involved in AVM development in ALK1-deficient (30) and endoglin-deficient (31) mice and in patients with HHT2 (36). Specifically, ribosomal protein S6 (S6) phosphorylation, a downstream effector of mTORC1 and Akt, was reported to be increased in the ECs of AVMs in the Alk1 ${ }^{\mathrm{i} E \mathrm{EC}}$ mouse retina and in BMP9- and BMP10-immunoblocked (BMP9/10ib) mice (30), another HHT model. In addition, administration of PI3K inhibitors, such as wortmannin, partially reduced retinal AVM numbers in $A l k 1^{\mathrm{i} \triangle \mathrm{EC}}, E^{\mathrm{E}} g^{\mathrm{i} \triangle \mathrm{EC}}$, and $\operatorname{Smad} 4^{\mathrm{i} \triangle \mathrm{EC}}$ mice $(25,30,31)$. Akt1 deficiency also mitigated AVM pathology in the Smad4 $4^{\mathrm{i} E \mathrm{EC}}$ model (25). Thus, overactivated VEGFR2 and PI3K/Akt/mTOR pathways are critical drivers - in addition to ALK1-endoglin loss of function - of HHT pathogenesis and AVM development.

Recently, we (32) and others (37) have reported that tacrolimus (Tac) was associated with Smad1/5/8 signaling activating properties, both in vitro in primary ECs and in vivo in mouse models of HHT and PAH. Spiekerkoetter et al. further reported in a cell-based $\mathrm{BMP} / \mathrm{Smad}$ transactivation assay and in human pulmonary arterial ECs that the mTOR inhibitor sirolimus (Siro, rapamycin), a macrolide analog of Tac, also activated Smad1/5/8, although less potently than Tac (37). These data suggest the intriguing possibility that Siro could both prevent mTOR overactivation and rescue Smad1/5/8 signaling in HHT models. In line with this hypothesis, a 2006 case study reported the potential beneficial effect of an immunosuppressive regimen, which contained Siro, in a probable HHT patient who underwent liver transplantation after high-output cardiac failure and hepatic AVM development (38). In this patient, the authors observed a reduction in angiodysplasia and mucosal hemorrhage. They proposed that the combination of Siro plus aspirin, in the antirejection regimen, could have contributed to the observed effects in this patient (38).

Several receptor tyrosine kinase (RTK) inhibitors that target VEGFR2 are FDA/EMA approved; one of these, pazopanib, showed beneficial effects in ALK1-deficient mice (39) and in a cohort of patients with HHT (40). A recent case study reported that nintedanib (Nin, BIBF 1120), a VEGFR2-targeting RTK inhibitor that is approved for idiopathic pulmonary fibrosis (IPF) treatment, produced marked reductions in epistaxis and skin telangiectasias in a patient affected by both HHT and IPF (41). Nin is of particular interest compared with other classical RTK inhibitors because it has a narrow spectrum of inhibition and is associated with minimal adverse effects.

In this context and in the current study, we sought to determine whether concurrent pharmacological targeting of Smad1/5/8 reduction, and of mTOR and VEGFR2 activation, could rescue the signaling defects and vascular pathology of HHT. We report that Siro and Nin, when administered in combination, mechanistically synergize at the transcriptional and signaling levels to efficiently prevent and reverse the vascular pathology, and associated bleeding and anemia, in 2 HHT mouse models.

\section{Results}

Siro and Nin combination treatment prevents vein dilation, hypervascularization, and AVM development in the retina of the $t B M P 9 / 10 i b$ mice. Recently, we reported the development of an HHT mouse model, which uses the transmammary route for administering BMP9- and BMP10-blocking antibodies to nursing mouse pups (22, 32 ), hereafter referred to as the transmammary-treated, BMP9/10immunoblocked (tBMP9/10ib) mice. We showed that this model, which has the advantage of being less invasive for the pups and more suitable for the analysis of large cohorts, leads to reduced ALK1 signaling and the development of a robust HHT-like pathology in the retinal vasculature of the pups $(22,32)$. In this model, we found that Tac significantly activated endothelial ALK1 signaling in vivo and prevented hypervascularization of the tBMP9/10ib retina. Further analyses of Tac efficacy in preventing vascular pathology in tBMP9/10ib mice revealed, however, a relatively modest effect of the drug on AVM development (32). These data prompted us to determine whether the macrolide analog of Tac, Siro (administered alone and in combination with Nin), is more potent in reducing the vascular pathology of the tBMP9/10ib retina.

Studies were conducted prior to these experiments to determine the appropriate dosing and injection schedule of the drugs in pups. The highest dose of Siro that did not affect normal vascular development was chosen $(0.5 \mathrm{mg} / \mathrm{kg}$, i.p. injection of the pups, assessed in the neonatal retina). Similarly, in order to prevent changes in retinal vascular development, we determined that Nin should be given every third day and not before P5. With this schedule for Nin injection, the highest dose of the drug that did not affect physiological vascular development was determined to be $0.3 \mathrm{mg} /$ $\mathrm{kg}$ (i.p., data not shown). Coadministration of Siro and Nin (Siro + Nin) at these dosing and injection schedules did not significantly affect normal vascular growth, indicating that physiological angiogenesis in the retina was not inhibited by the drug combination (Supplemental Figure 1; supplemental material available online with this article; https://doi.org/10.1172/JCI127425DS1). At this 
A

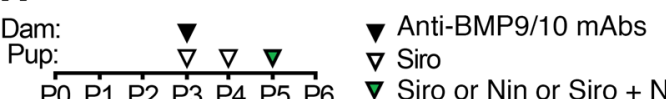

tBMPg/10ib
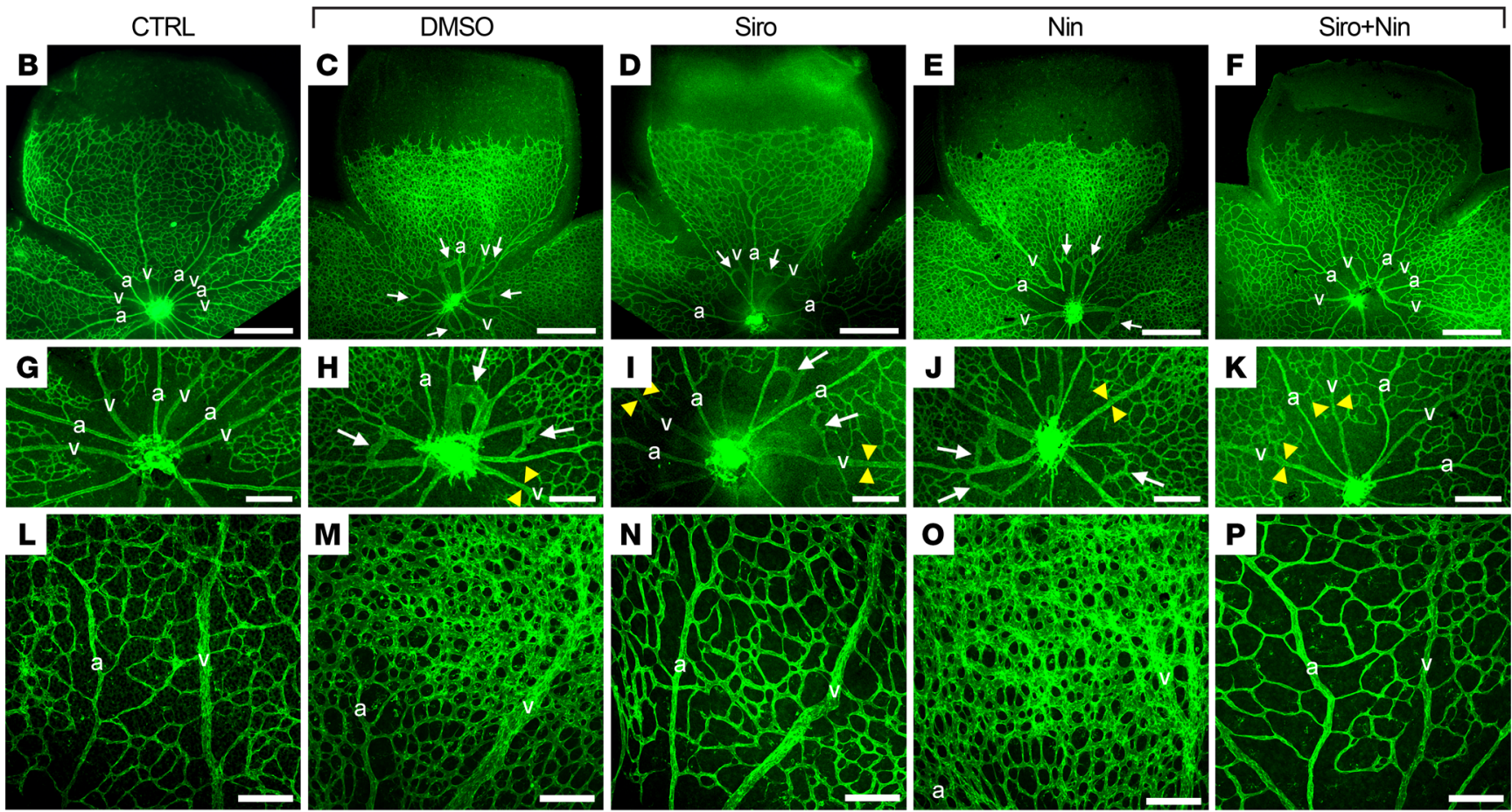

Q

$\mathbf{R}$
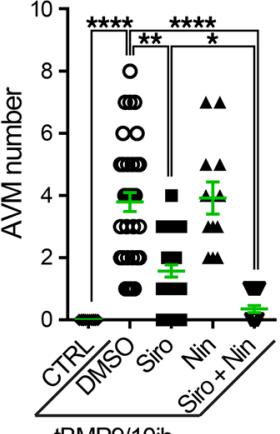

tBMP9/10ib

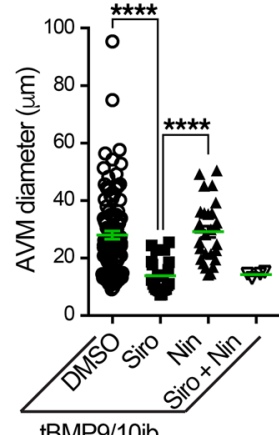

S

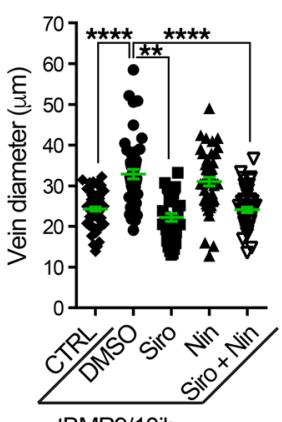

$\mathbf{T}$

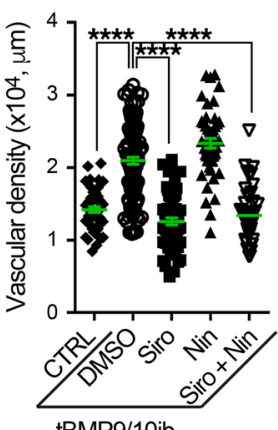

tBMP9/10ib

Figure 1. Siro + Nin prevents vein dilation, hypervascularization, and AVMs in the tBMPg/10ib mouse retina. (A) Schematic representation of the protocol of disease induction (i.p. injections of the lactating dams with anti-BMPg/10 antibodies, anti-BMPg/10 mAbs) and of drug treatments (i.p. injections of the pups with Siro and/or Nin). Arrowheads indicate the postnatal days of injection. Control (CTRL) mouse pups were obtained by injecting the lactating dams with isotype controls and by directly injecting them with DMSO. Pups were euthanized at P6. (B-P) Representative images of retinas stained with fluorescent isolectin B4 (green) from CTRL (B, G, and L) mice and tBMP9/10ib mice treated with vehicle (DMSO; C, H, and M), Siro (D, I, and N), Nin (E, J, and $\mathbf{0}$ ), or the combination of Siro and $\operatorname{Nin}(\mathbf{F}, \mathbf{K}$, and $\mathbf{P})$. Higher magnification in $\mathbf{G}-\mathbf{K}$ and $\mathbf{L}-\mathbf{P}$ shows respectively retinal vein diameter (yellow arrowheads) and retinal vascular fields (plexus area) between an artery (a) and a vein (v). Arrows denote AVMs. Scale bars: $500 \mu \mathrm{m}(\mathbf{B}-\mathbf{F}), 200 \mu \mathrm{m}$ (G-K), and $100 \mu \mathrm{m}$ (L-P). (Q-T) Scatter plots measuring AVM number (Q), AVM diameter (R), vein diameter (S), and vascular plexus density (T) in CTRL and tBMP9/10ib mice treated as in B-F. Data represent individual retinas and mean \pm SEM $(n=14,38,28,12$, and 20 retinas for the CTRL, DMSO, Siro, Nin, and Siro + Nin groups, respectively); Kruskal-Wallis test, post hoc Dunn's multiple-comparisons test. ${ }^{*} P<0.05 ;{ }^{* *} P<0.01 ;{ }^{* * *} P<0.0001$.

dosing, liquid chromatography/mass spectrometry (LC-MS) analyses measured average serum concentrations of $9.0 \mathrm{nM}$ Siro and $5.3 \mathrm{nM}$ Nin in the injected P6 pups.

As shown in Figure 1A and described previously $(22,32)$, vascular pathology in pups was initiated at P3 by 1 i.p. injection of the dams with anti-BMP9/10 antibodies. Mouse pups were administered preventively and daily with Siro from P3 to P5 (0.5 mg/kg, i.p.) and with Nin at P5 (0.3 mg/ $\mathrm{kg}$, i.p.). Mice were then analyzed at P6 (Figure 1A), a time point at which retinal vessel dilation, hypervascularization, and AVMs can readily be observed and quantified in this model $(22,32)$. In the tBMP9/10ib retinas, Siro significantly reduced AVM number 


\section{A}

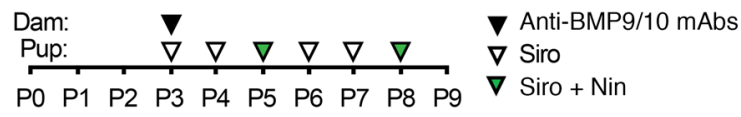

B

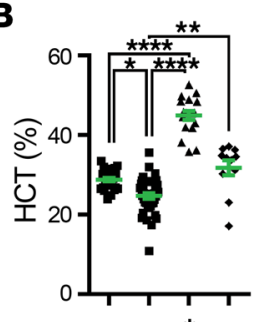

C
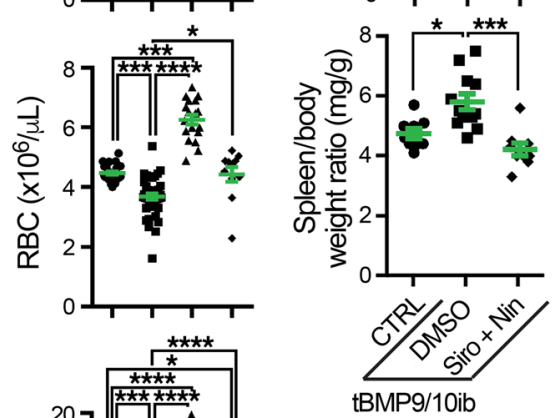

D
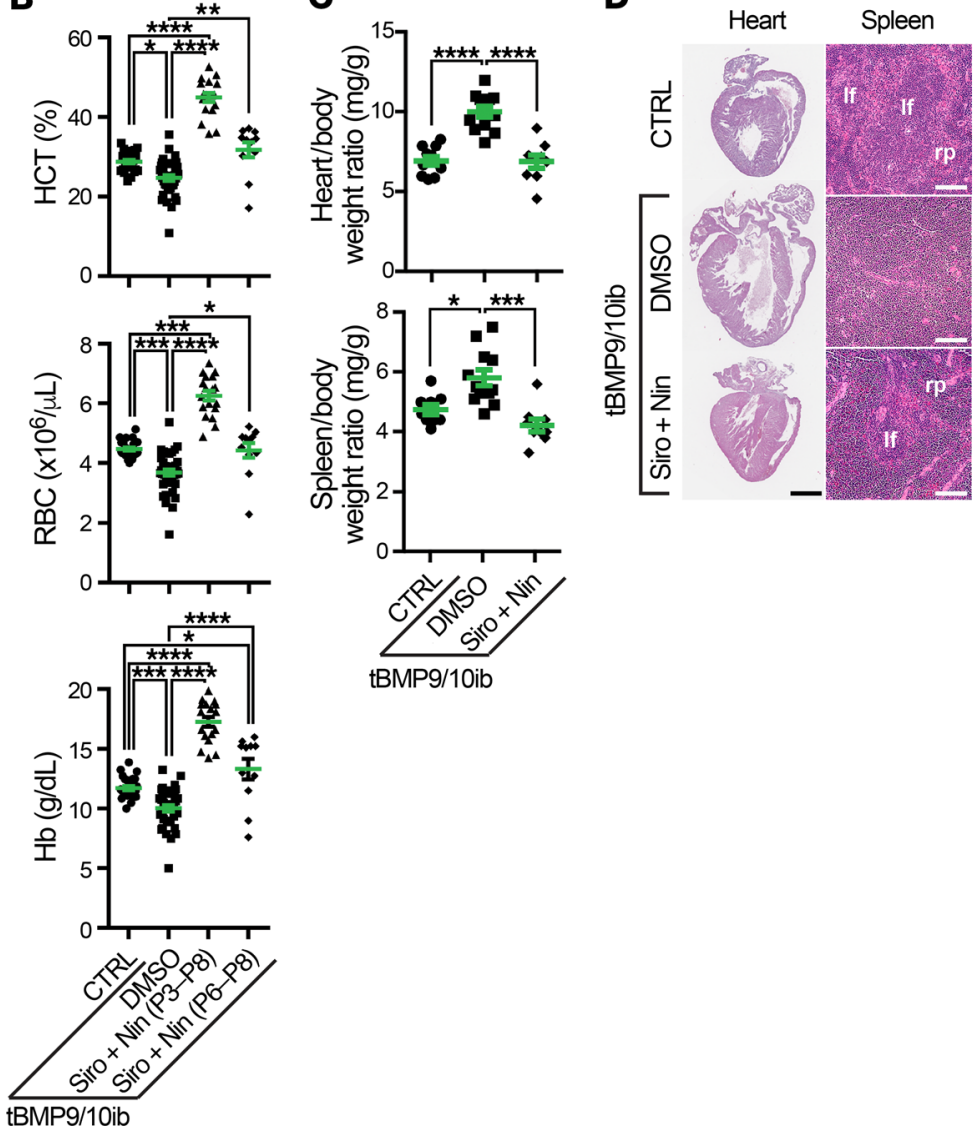

Figure 2. Siro + Nin prevents anemia in tBMPg/10ib mice. (A) Schematic representation of the protocol for disease induction and drug treatments. Pups were euthanized at P9. (B) Scatter plots measuring hematocrit (HCT) level, red blood cell (RBC) number, and hemoglobin $(\mathrm{Hb})$ level in $\mathrm{Pg}$ control (CTRL) and tBMPg/10ib mice treated with Siro + Nin at P3-P8 (as in A) or P6-P8 (see Figure 4A). Data represent individual mice and mean \pm SEM ( $n=26-29,35-40,18,11$ mice for the CTRL, DMSO, Siro + Nin [P3-P8], and Siro + Nin [P6-P8] groups, respectively); HCT and RBC analyses: Kruskal-Wallis test, post hoc Dunn's multiple-comparisons test; Hb analysis: 1-way ANOVA, Tukey's multiple-comparisons test. CBC parameters of the controls were within the expected ranges for $[57 \mathrm{BL} / 6$ ) neonates. (C and D) P9 mouse heart and spleen analyses of CTRL and tBMPg/10ib mice treated as in A. Heart/body and spleen/body weight ratios are shown in C. Data represent individual mice and mean \pm SEM $(n=9-11,12$, and 9 mice for the CTRL, DMSO, and Siro + Nin groups, respectively); 1-way ANOVA, Tukey's multiple-comparisons test. ${ }^{*} P<0.05$; ${ }^{* *} P<0.01 ;{ }^{* *} P<0.001 ;{ }^{* * *} P<0.0001$. Heart and spleen sections stained with H\&E are shown in $\mathbf{D}$. If, lymphoid follicle; rp, red pulp. Scale bars: $1 \mathrm{~mm}$ (D, heart), $10 \mu \mathrm{m}$ (D, spleen).

and AVM diameter (Figure 1, B-D, G-I, Q, and R). In addition, as we observed previously for Tac at the same dosing $(0.5 \mathrm{mg} / \mathrm{kg}$, P3-P5, i.p.) (32), Siro prevented vein dilation (Figure 1S) and the increase in density of the vascular plexus (Figure 1, B-D, L-N, and $\mathrm{T}$ ). In contrast, Nin at the tested dosing failed to reduce any of the investigated vascular defects of the tBMP9/10ib retinas (Figure 1, B, C, E, G, H, J, L, M, O, and Q-T).

Although Siro treatment was able to significantly reduce AVM number and size, its preventive effect was only partial
(AVM number, mean $=3.79 \pm 0.30$ in DMSO-treated tBMP9/10ib retinas vs. mean $=1.57 \pm 0.19$ in Sirotreated tBMP9/10ib retinas, $P \leq 0.01$ ). Knowing that evidence is strong to suggest that VEGFR2 signaling is increased in HHT models and that Siro demonstrated no efficacy in inhibiting VEGFR2-mediated MAPK signaling activation in primary ECs (Supplemental Figure 2 and ref. 42), we asked whether the VEGFR2 inhibitor Nin could increase Siro potency in preventing AVMs. Combination treatment with the 2 drugs resulted in a significant increase in Siro's anti-AVM effect (Figure 1, B-K and Q; AVM number after treatment, mean $=0.35 \pm 0.11, P \leq$ 0.0001 vs. DMSO-treated tBMP9/10ib retinas, and $P \leq$ 0.05 vs. Siro-treated tBMP9/10ib retinas). The Siro + Nin combination did not further increase the effect of Siro on vein dilation and vascular density, as Siro alone was sufficient to fully correct these 2 defects (Figure 1, L-P, S, and $\mathrm{T})$. Measurement of the diameter of the few remaining AVMs identified in the retina of the Siro + Nin-treated mice also revealed no difference compared with treatment with Siro alone (Figure 1R). Together, these data in tBMP9/10ib mice show that Siro fully normalized vein dilation and hypervascularization, and significantly lowered AVM number and size in the retina. Furthermore, and more strikingly, Nin significantly strengthened the anti-AVM effect of Siro.

Siro + Nin prevents anemia and retinal bleeding in tBMP9/10ib mice. We next assessed pathology progression in tBMP9/10ib pups at P9. As before, vascular pathology in pups was initiated at P3 by 1 i.p. injection of the dams with anti-BMP9/10 antibodies (Figure $2 \mathrm{~A})$. Complete blood count $(\mathrm{CBC})$ revealed significant reductions in hematocrit level, red blood cell (RBC) number, and hemoglobin level (Figure 2B), indicative of anemia in P9 tBMP9/10ib pups. Furthermore, severe cardiomegaly (Figure 2, C and D) and splenomegaly (Figure 2C) developed in tBMP9/10ib mice. Splenomegaly was accompanied by an expansion of the red pulp (Figure 2D), indicating the presence of splenic erythropoietic stress response consecutive to anemia. These data prompted us to investigate whether tBMP9/10ib mice were actively bleeding. Inspection of the retinas using whole-mount IHC with an antibody directed against the RBC marker Ter119 revealed the presence of strongly immunoreactive patches in multiple areas of the tBMP9/10ib retinas (Figure 3, A, B, and $\mathrm{M}$ ). Single-cell resolution confocal analyses and $3 \mathrm{D}$ reconstruction showed the presence of $\mathrm{RBC}$ patches outside the tBMP9/10ib retinal vasculature (Figure 3, D-F and G-I, and animation in Supplemental Video 1). Interestingly, isolectin B4-positive projections could clearly be identified near the center of some of these RBC accumulations, suggesting that they might represent transversal vascular projections at the origin of the bleeding (Figure 3, G-I, arrows, and Supplemental Video 1). Treatment of the tBMP9/10ib mice with Siro + Nin from P3 to P8 (Figure 2A) significantly reduced the area occupied by retinal bleeding (Figure 3, A-M) and fully prevented 


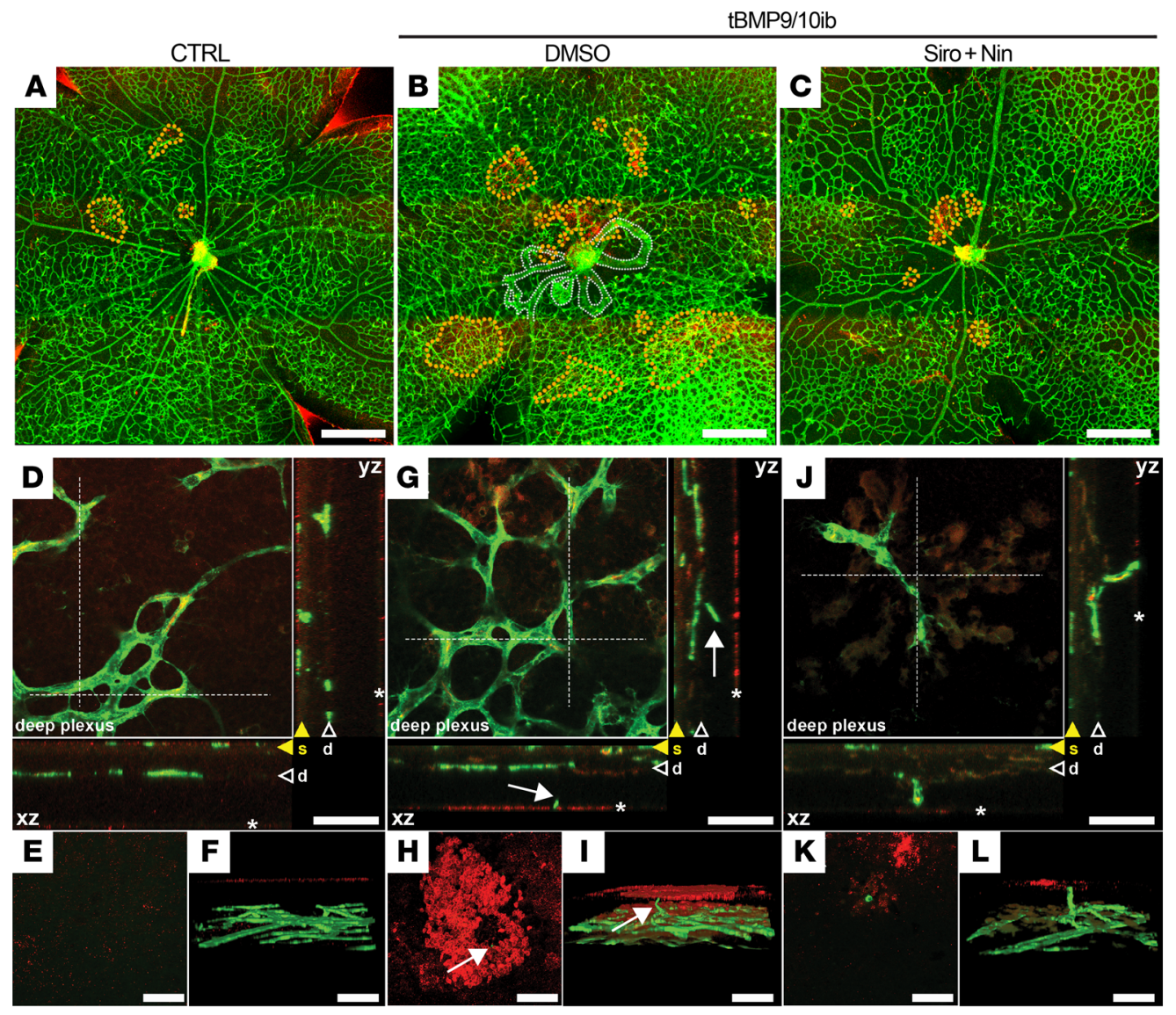

M

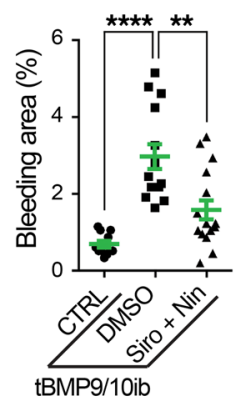

$\mathbf{N}$

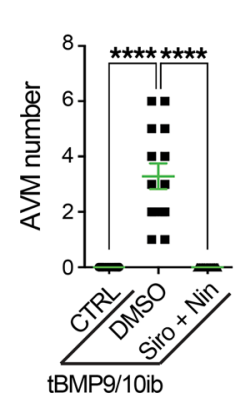

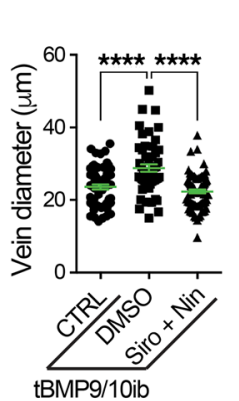

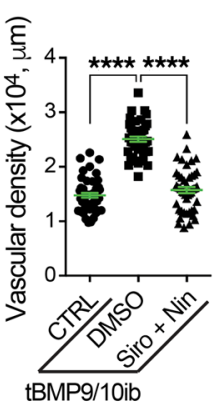

Figure 3. Siro + Nin prevents retinal bleeding in tBMP9/10ib mice. (A-L) Representative images showing microbleeds (dotted orange lines in A-C) in retinas stained with fluorescent isolectin B4 (green) and anti-Ter119 antibody (red) from control (CTRL; $\mathbf{A}$ and $\mathbf{D}-\mathbf{F}$ ) and tBMP9/10ib mice treated with DMSO (B and $\mathbf{G}-\mathbf{I})$ or Siro + Nin (C and $\mathbf{J}-\mathbf{L})$, as in Figure 2A. Dotted white lines in $\mathbf{B}$ indicate AVMs. Higher magnifications in $\mathbf{D}-\mathbf{L}$ show the extending deeper retinal plexus (arrowheads marked as "d" and orthogonal view [xz and yz planes] of the stack of images at the level of the dotted lines are shown in $\mathbf{D}$, G, and J). Orthogonal views in $\mathbf{D}, \mathbf{G}$, and J show superficial vascular plexus (yellow arrowheads marked as "s"), vascular branches projecting to the outer layer of the retina (white arrows), and microbleeds (asterisks). Focal plane views $(\mathbf{E}, \mathbf{H}$, and $\mathbf{K}$ ) and $3 \mathrm{D}$ reconstructions $(\mathbf{F}, \mathbf{I}$, and $\mathbf{L})$ identify microbleeds. Scale bars: $500 \mu \mathrm{m}(\mathbf{A}-\mathbf{C})$ and $50 \mu \mathrm{m}$ (D-L). (M) Scatter plot measuring bleeding areas, expressed as a percentage of the retinal vascular area occupied by extravascular accumulation of RBCs in mice treated as in Figure 2A. Data represent mean \pm SEM $(n=6,4,4$ mice for the CTRL, DMSO, and Siro + Nin groups, respectively); 1-way ANOVA, Tukey's multiple-comparisons test. (N) Scatter plots measuring AVM number, vein diameter, and vascular plexus density in the retina of P9 pups treated as in Figure 2A. Data represent individual retinas and mean \pm SEM $(n=7,7$, and 8 mice for the CTRL, DMSO, and Siro + Nin groups, respectively); AVM number and vein diameter analyses: 1-way ANOVA, Tukey's multiple-comparisons test; vascular density analysis: KruskalWallis test, post hoc Dunn's multiple-comparisons test. ${ }^{* *} P<0.01 ;{ }^{* * *} P<0.0001$.

the decrease in hematocrit level, RBC number, and hemoglobin level (Figure 2B), as well as blocked cardiomegaly, splenomegaly, and the loss of splenic architecture (Figure 2, C and D). Thus, Siro + Nin combination treatment prevented anemia and retinal bleeding in tBMP9/10ib mice.
Because evidence is mounting that AVM development is controlled by blood flow (43) and because P9 tBMP9/10ib mice display significant cardiomegaly, we asked (a) whether tBMP9/10ib mice develop cardiac defects leading to higher cardiac output and (b) whether the drugs could indirectly reduce vascular pathology 

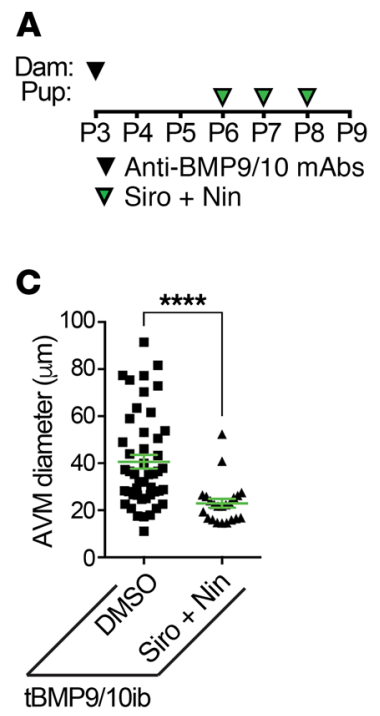

B

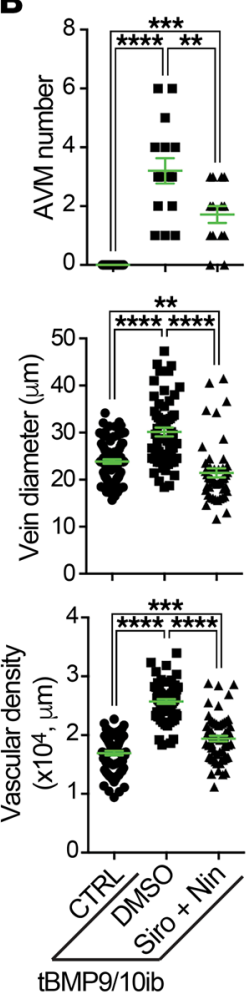

Figure 4. Siro + Nin reverses retinal vascular pathology in tBMP9/10ib mice. (A) Schematic representation of the protocol for disease induction and drug treatments. Pups were euthanized at P9. (B and C) Scatter plots measuring AVM number, vein diameter, vascular plexus density (B), and AVM diameter (C) in the retina of CTRL and tBMP9/10ib mice treated as in A. Data represent individual retinas and mean $\pm \operatorname{SEM}(n=9,8$, and 7 mice for the CTRL, DMSO, and Siro + Nin groups, respectively); AVM number and vascular density analyses: 1-way ANOVA, Tukey's multiple-comparisons test; AVM diameter analysis: Mann-Whitney test; vein diameter analysis: Kruskal-Wallis test, post hoc Dunn's multiple-comparisons test; ${ }^{*} P<$ $0.01 ;{ }^{* *} P<0.001 ;{ }^{* * *} P<0.0001$.

by correcting these cardiac defects. Although a modest decrease in heart rate was measured by Doppler ultrasonography upon drug treatment in tBMP9/10ib mice, heart rate was overall not significantly changed in DMSO-treated and Siro + Nin-treated tBMP9/10ib pups compared with normal pups (Supplemental Figure 3A). In addition, no significant defects in cardiac output (Supplemental Figure 3B) or pulmonary arterial pressure (Supplemental Figure 3C) measures were found between all groups. These data demonstrate that basic cardiac function is normal in tBMP9/10ib pups, at least until P9, and that Siro + Nin combination treatment is therefore unlikely to act on the vasculature by changing cardiac output.

Siro + Nin reverses vascular pathology in tBMP9/10ib mice. We next analyzed the retinal vasculature of P9 tBMP9/10ib mice treated or not with Siro + Nin. On average, approximately 4 AVMs were detected in tBMP9/10ib mice (Figure 3N and Supplemental Figure 4), indicating that no additional AVMs developed between P6 (mean $=3.79 \pm 0.30$, Figure $1 \mathrm{Q}$ ) and P9. Strikingly, Siro + Nintreated P6 mice still contained some AVMs $(n=0.35 \pm 0.11$, Figure 1Q), but the P9 tBMP9/10ib mouse retinas that were treated with the drugs for 3 additional days were devoid of AVMs (Figure

$3 \mathrm{~N}$ and Supplemental Figure 4). In addition, the 6-day Siro + Nin treatment (P3-P8, Figure 2A), as we observed after the 3-day Siro + Nin treatment (P3-P5, Figure 1), fully prevented vein dilation and hypervascularization (Figure $3 \mathrm{~N}$ and Supplemental Figure 4). LC-MS analyses measured an increase in average drug concentrations in the pup serum between P6 and P9: from $9.0 \mathrm{nM}$ to 22.6 $\mathrm{nM}$ Siro and from $5.3 \mathrm{nM}$ to $24.7 \mathrm{nM}$ Nin, indicating that 3 additional days of drug treatment led to an accumulation of the drugs in the circulation.

These data suggest that Siro + Nin treatment might also reverse existing AVMs, since some AVMs disappeared between P6 and $\mathrm{P} 9$. To directly address this possibility, we implemented a protocol that started the drug treatment after retinal vascular pathology was established. Specifically, pathology was induced as before at P3 and pups were then treated at P6 with Siro + Nin, a time point where we have established the presence of robust vein dilation, hypervascularization, and of approximately 4 AVMs per retina (Figure 1). Pups were treated for 3 days, from P6 to P8 (P6-P8), and analyzed at P9 (Figure 4A). We found that Siro + Nin administered after pathology induction significantly reduced overall vascular pathology (Supplemental Figure 5), including AVM number, AVM size, vein dilation, and vascular density (Figure 4, B and C). In addition, P6-P8 Siro + Nin treatment significantly increased hematocrit level, RBC number, and hemoglobin level in anemic tBMP9/10ib mice (Figure 2B).

Because disease induction was triggered by only 1 i.p. injection at P3 of anti-BMP9/10 antibodies, we verified that the observed effects of Siro + Nin in P9 pups were not facilitated by a disappearance of the disease-causing anti-BMP9/10-blocking antibodies from the pup circulation. Using specific anti-BMP9 and anti-BMP10 antibody ELISAs, we found that serum antibody concentrations were stable between $\mathrm{P} 6$ and P9, and reached approximately $70 \mu \mathrm{g} / \mathrm{mL}$ for the anti-BMP9 antibody and approximately $85 \mu \mathrm{g} / \mathrm{mL}$ for the anti-BMP10 antibody (Supplemental Figure 6). Thus, the disease-causing effects of the anti-BMP9/10 antibodies were maintained between P6 and P9, and therefore, the drug combination effect on preexisting AVMs occurred in a maintained pathogenic environment. Taken together, these findings demonstrate that Siro + Nin combination treatment not only prevented, but also reversed, the retinal vascular pathology of the tBMP9/10ib mice.

Siro + Nin prevents vascular pathology in the oral mucosa and lungs of the $t B M P 9 / 10 i b$ mice. The oral mucosa and lungs are major sites of vascular lesion development in patients with HHT. We investigated whether vascular defects were observed in these tissues of the P9 tBMP9/10ib mice. Injections of latex blue dye in the blood circulation were used to visualize vascular pathology. In the tBMP9/10ib mouse tongue and palate, mucosal vein dilation and hyperproliferative vascular defects were clearly identified after latex dye injection (Figure 5, A, B, D, and E). Significant enlargements of the lingual and greater palatine vessels could be measured compared with control tongues and palates (Figure 5J). In the lungs, the dye invaded a hypervascularized network of dilated small vessels throughout the lobar system and revealed an enlargement of the main pulmonary vessels of the tBMP9/10ib mice (Figure 5, G, H, and J). Siro + Nin treatment of the tBMP9/10ib mice significantly and efficiently prevented the 


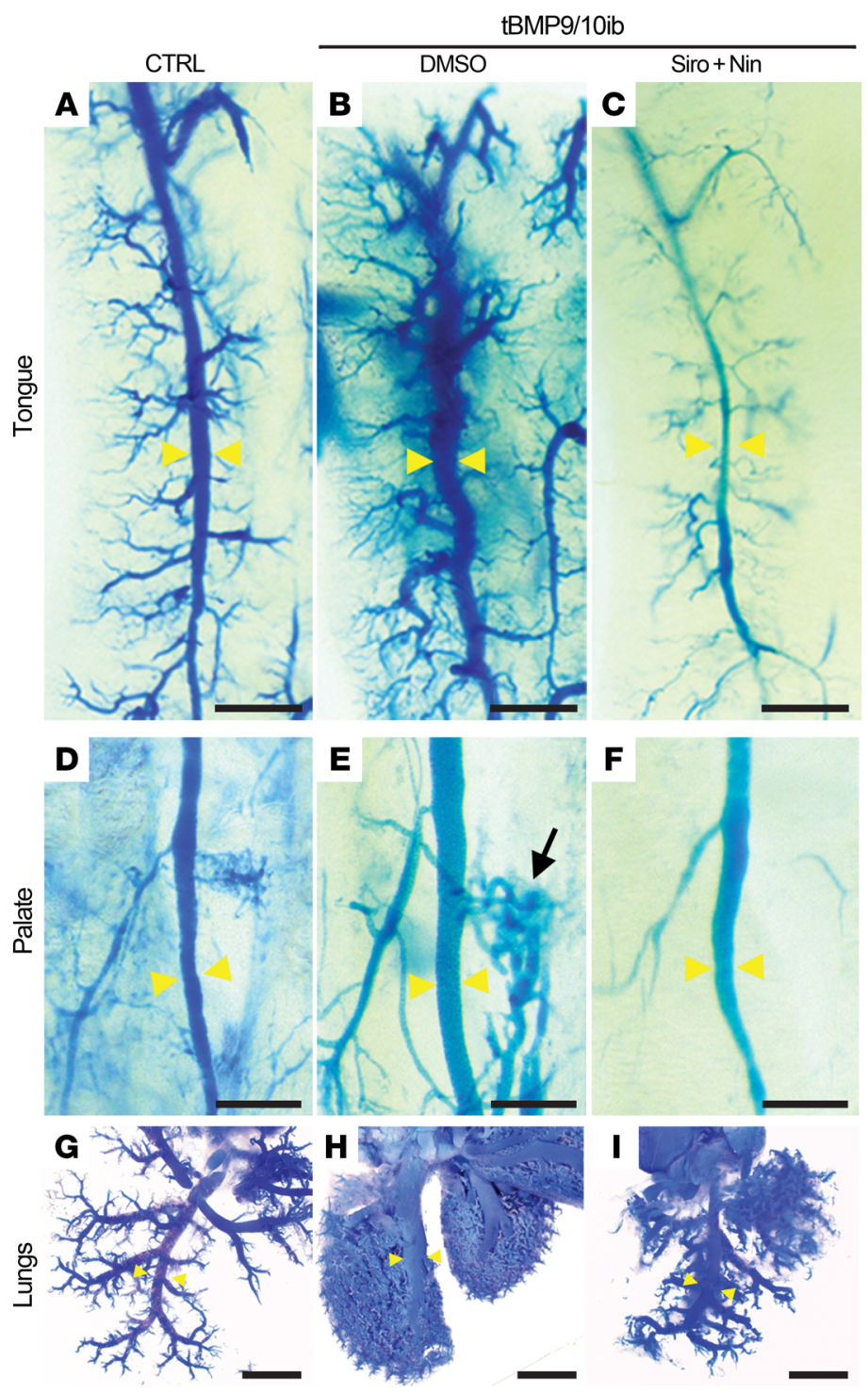

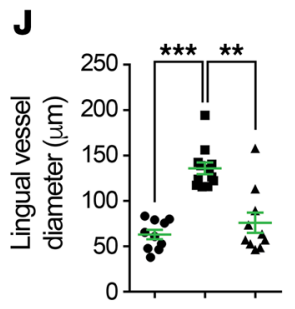
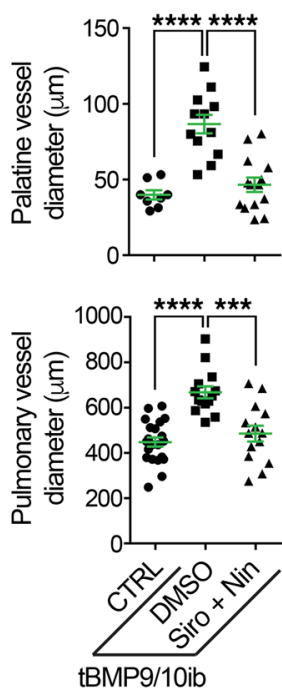

Figure 5. Siro + Nin prevents vascular pathology in the oral mucosa and lungs of the tBMP9/10ib mice. (A-I) Representative images of latex blue dye-perfused mouse tongue, palate, and lungs of control (CTRL; A, D, and $\mathbf{G}$ ) and tBMPg/10ib mice treated with DMSO (B, E, and $\mathbf{H})$ or Siro $+\operatorname{Nin}(\mathbf{C}, \mathbf{F}$, and I), as in Figure 2A. Yellow arrowheads indicate lingual, palatine, and pulmonary vessels used for diameter measurements; black arrow denotes a dilation of a lateral branch in the palatine vasculature (E). Scale bars: $500 \mu \mathrm{m}(\mathbf{A}-\mathbf{F})$ and $2 \mathrm{~mm}$ (G-I). (J) Scatter plots measuring lingual, palatine, and pulmonary vessel diameter. Data represent mean $\pm \mathrm{SEM}$; lingual vessel diameter analysis $(n=3,4$, and 3 mice for the CTRL, DMSO, and Siro + Nin groups, respectively): Kruskal-Wallis test, post hoc Dunn's multiple-comparisons test; palatine ( $n=2,3$, and 4 mice) and pulmonary ( $n=10,6$, and 6 mice) vessel diameter analyses: 1-way ANOVA, Tukey's multiple-comparisons test. ${ }^{*} P<0.01$; ${ }^{* * *} P<0.001 ;{ }^{* * *} P<0.0001$. hyperproliferative vascular pathology and vessel dilation phenotype observed in the tongue, palate mucosa, and lungs (Figure 5). Thus, Siro + Nin combination treatment reduced vascular pathology in the oral mucosa and lungs.

Siro + Nin treatment corrects a gene expression signature and prevents vascular pathology in the liver of the $\mathrm{BBMP} 9 / 10 \mathrm{ib}$ mice. The liver is the most vascularized organ of the body and is a major site of vascular lesion development in patients with HHT, more specifically HHT2 (44). We next investigated whether vascular defects could be observed in the livers of the P9 tBMP9/10ib mice. Vascular pathology induction and drug treatments in pups were performed as above (Figure 2A). Latex dye tissue invasion was enhanced in the tBMP9/10ib mouse livers and revealed a significant enlargement of the hepatic vessels (Figure 6, A, B, and D). H\&E staining showed the presence of marked local liver injury, characterized by the presence of significant hepatocyte vacuolation and hepatocellular necrosis (Figure 6, E, F, H, and I, and Supplemental Figure 7), a pathology that could result from ischemic events.

To gain insight into the mechanism of liver injury, we performed a proteome array for angiogenesis-related factors. This screen identified plasminogen activator inhibitor 1 (PAI-1) as a protein strongly upregulated in the tBMP9/10ib mouse livers compared with livers from control mice (Figure 6K). PAI-1 is of interest because it is upregulated during hypoxia (45), and might thus represent a response to ischemia. PAI-1 elevation in the tBMP9/10ib mouse livers was confirmed by Western blot (Figure 6L) and IHC (Figure 6, M, N, and P) analyses. To verify that hypoxia was occurring in the tBMP9/10ib mouse livers, tissue sections were stained for hypoxia-inducible factor $1 \alpha$ (HIF-1 $\alpha$ ), a transcriptional factor marker and master regulator of hypoxia (46). We found a strong upregulation of liver HIF-1 $\alpha$ expression in tBMP9/10ib mice compared with control mice (Figure 6, Q, R, and T). These data show that tBMP9/10ib mice develop a robust vascular pathology in the liver. Strikingly, Siro + Nin treatment of the tBMP9/10ib mice significantly prevented the hyperproliferative vascular pathology and vessel dilation phenotype of the liver (Figure 6, A-D). In addition, Siro + Nin efficiently reduced hepatocyte vacuolation and necrosis (Figure 6, E-J, and Supplemental Figure 7), as well as prevented the overexpression of PAI- 1 and HIF-1 $\alpha$ in the liver of the tBMP9/10ib mice (Figure 6, M-T). Together, these results demonstrate that 

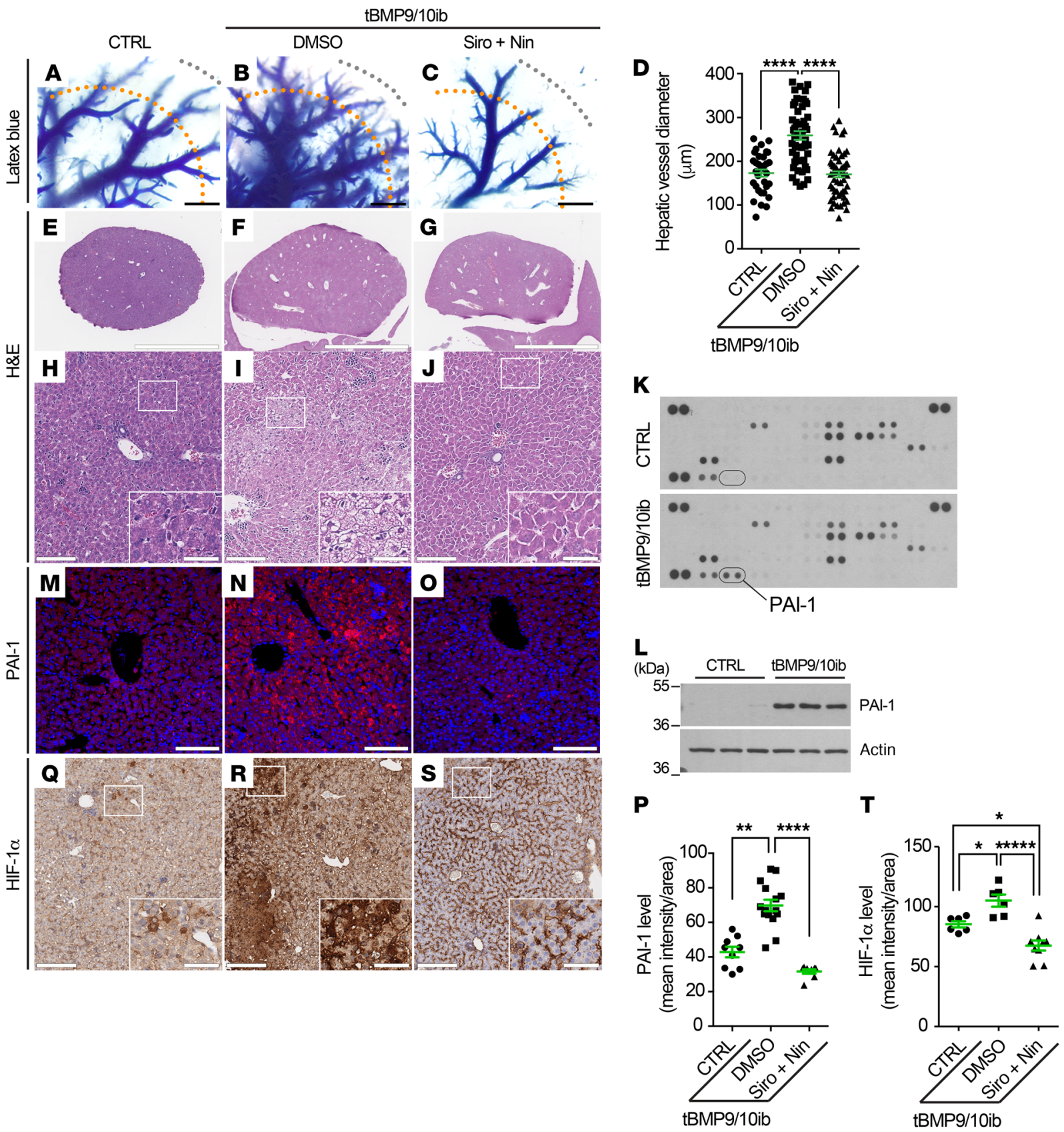

Figure 6. Liver vascular pathology and liver disease in tBMP9/10ib mice: Effects of Siro + Nin treatment. (A-C) Latex blue dye-perfused livers from tBMP9/10ib mice treated with vehicle (DMSO; B) or Siro + Nin (C), and control mice treated with DMSO (CTRL; A), as in Figure 2A. (D) Scatter plot measuring hepatic vessel diameter. Diameters of these vessels (most likely arterial) were measured at 2,000 $\mu \mathrm{m}$ (orange dotted lines) from the margin of the liver (gray dotted lines). Data represent mean \pm SEM ( $n=4$ livers per condition); Kruskal-Wallis test, post hoc Dunn's multiple-comparisons test. (E-J) Liver sections stained with H\&E at low magnification (E-G) and high magnification (H-J) from tBMP9/10ib and CTRL mice treated as in $\mathbf{A}-\mathbf{C}$; insets in $\mathbf{H}-\mathbf{J}$ indicate magnified areas. (K-0) PAI-1 immunodetection by proteomic array (K, pooled homogenates from $n=3$ mice) and Western blot (L, $n=3$ mice) of liver homogenates, and by immunofluorescence staining in liver sections (M-0) from mice treated as in $\mathbf{A}-\mathbf{C}$. (P) Scatter plot measuring PAI-1 levels, expressed as a mean intensity of fluorescence per sampled area. Data represent mean \pm SEM ( $n=3$ livers per condition); Kruskal-Wallis test, post hoc Dunn's multiple-comparisons test. (Q-S) HIF-1 $\alpha \mathrm{IHC}$ in liver sections from mice treated as in $\mathbf{A}-\mathbf{C}$; insets indicate magnified areas. Histology data are representative of at least 3 independent mice/condition. Scale bars: $1000 \mu \mathrm{m}$ (A-C), $3 \mathrm{~mm}(\mathbf{E}-\mathbf{C}), 100 \mu \mathrm{m}$ (H-J, M-0, and Q-S), $35 \mu \mathrm{m}$ in insets (H-J and Q-S). (T) Scatter plot measuring HIF-1 $\alpha$ levels, expressed as a mean intensity of fluorescence per sampled area. Data represent mean \pm SEM ( $n=3$ livers per condition); 1 -way ANOVA test, post hoc Tukey's multiple-comparisons test. ${ }^{*} P<0.05 ;{ }^{* *} P<0.01 ;{ }^{* * *} P<0.0001$.

Siro + Nin combination treatment prevented vascular pathology in the liver and blocked liver disease in $\mathrm{BBMP} 9 / 10 \mathrm{ib}$ mice.

To further test the therapeutic potential of the Siro + Nin combination, we determined (a) whether gene expression changes could be detected in the tBMP9/10ib whole-liver tissue and (b) whether treatments with the 2 drugs (administered alone or in combination) could correct these transcriptomic changes. When we plotted the transcript expression changes (measured as log fold 
A

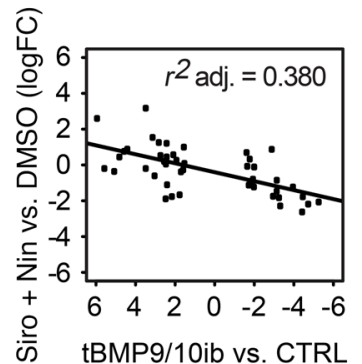

$(\log \mathrm{FC})$
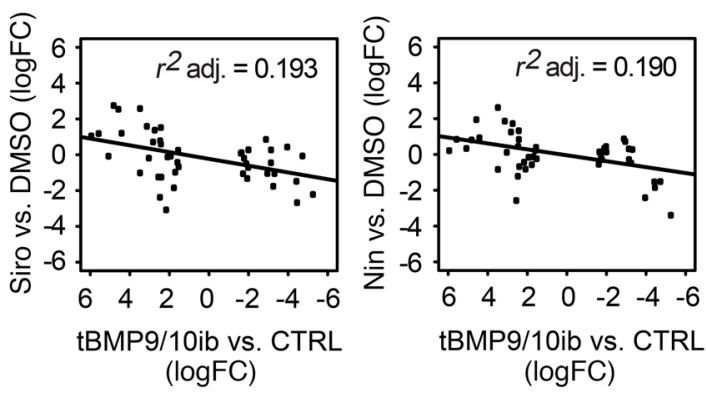

B

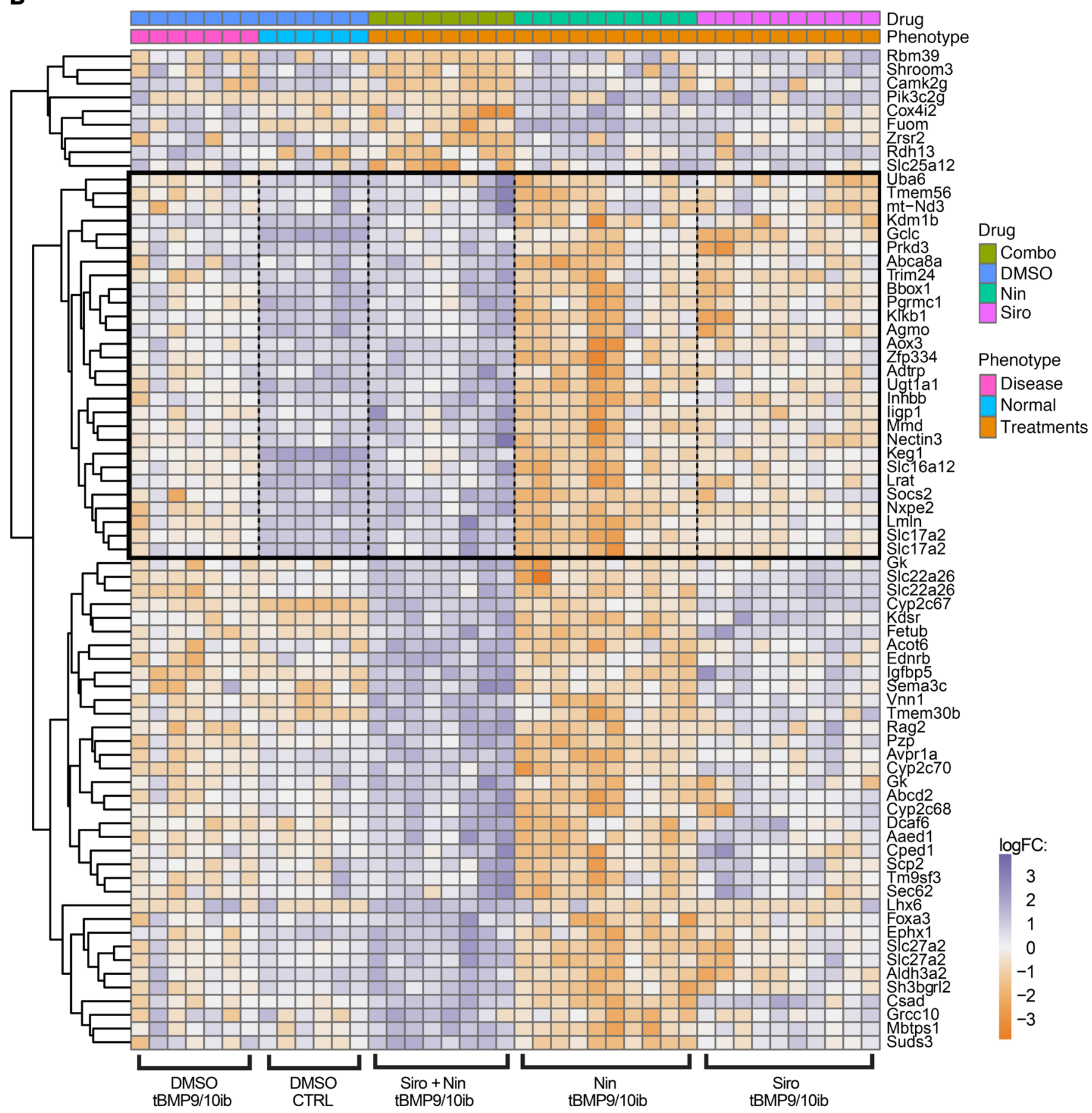


Figure 7. Siro + Nin corrects a gene expression signature detected in the tBMP9/10ib mouse liver. (A) Scatter plots comparing the liver gene expression changes (logFC, log fold change) observed upon treatment with Siro + Nin or individual drug (Siro or Nin), to the liver gene expression logFCs observed between tBMPg/10ib and normal control (CTRL) mice. Treatments were as in Figure 2A. The negative slope of the plots indicates that each treatment contributed to normalizing the liver gene expression changes seen in tBMPg/10ib versus CTRL mice. Correlation coefficients $\left(r^{2}\right)$ indicate a more robust normalization with the drug combination than with individual drugs. (B) Heatmap of liver transcripts synergistically differentially expressed by the drug combination. We identified the genes that responded to the drug combination differently than would be expected, assuming an additive effect of both drugs. A noninteracting drug would produce a COMBO expression equal to the average of the gene expression measured with each treatment (NIN or SIRO). The significant effects that we detected, measured with the statistical contrast COMBO - (NIN $+\mathrm{SIRO}) / 2$, produced gene expression changes that deviate from this average independent effect expectation. Transcripts were selected with FDR $\leq 0.5 \%$, log average expression $>-5$. For reference, liver transcripts in CTRL mice are shown on the heatmap. Gene expression in CTRL mice was used for normalization, but was not used in the selected transcripts that displayed synergistic drug response.

change values, logFC) in tBMP9/10ib pups versus normal controls, against the transcript expression $\log F C$ s obtained after treating the tBMP9/10ib pups with Siro + Nin versus vehicle (DMSO), an inverse correlation was found for Siro + Nin treatment $\left(r^{2}=\right.$ $0.380, P<0.001$; Figure 7A). An inverse correlation indicates that Siro + Nin treatment normalized some of the changes observed in the disease model (tBMP9/10ib pups) compared with normal controls. When the same comparison was done for Siro and Nin administered alone, a weaker correlation was measured $\left(r^{2}=0.193\right.$ and 0.190, respectively, $P<0.001$; Figure 7A). These results indicate that Siro + Nin combination better corrected the expression changes of liver transcripts detected in the tBMP9/10ib mice than either drug administered alone.

To illustrate the synergistic effect of Siro and Nin treatments on transcript expression, we identified transcripts that were differentially changed by the drug treatments in the tBMP9/10ib liver $(n$ = 6-9 biological replicates/group, FDR $\leq 0.5 \%$, log average expression $>-5$; Figure 7B, Supplemental Figure 8 and Supplemental Table 1). The black box in the heatmap of Figure 7B shows a subset of transcripts that were (a) significantly changed by Siro + Nin; (b) not changed by either Nin or Siro administered in isolation; and (c) whose expression was normalized, as compared with normal controls. This subset of transcripts confirms that a synergistic effect exists between Siro and Nin treatments at the gene expression level, and that this effect could normalize a subset of deregulated transcripts in the tBMP9/10ib liver. A functional enrichment analysis of the identified subset of normalized transcripts did not identify genes already reported to be involved in HHT or AVMs, but identified instead a network of genes involved in cell and protein metabolism (e.g., Uba6, Tmem56, mt-Nd3, Kdm1b, Gclc, Prkd3; GeneMANIA, ref. 47), a response indicative of strong and predominant gene expression changes consecutive to liver injury and deregulations in cell homeostasis.

Together, these transcriptomic data show that Siro and Nin treatments synergized and partially opposed a deregulated gene expression signature detected in the tBMP9/10ib liver. These results are important because they confirm the interaction and combination efficacy of the 2 drugs in reducing overall vascular pathology in tBMP9/10ib mice.

Siro + Nin reduces GI bleeding and anemia in adult Alk1-iKO mice. The effect of the drug combination was evaluated in an adult Alk1 inducible knockout (iKO) mouse model (R26 ${ }^{\mathrm{CreER} /+} A l k 1^{2 \mathrm{f} / 2 \mathrm{f}}$ ). In this model, ALK1 deficiency was induced by tamoxifen administration to generate severe GI bleeding and anemia in 9 days (Figure 8 and refs. 39, 48). Daily treatment with the same doses of Siro + Nin used for the tBMP9/10ib mice $(0.5 \mathrm{mg} / \mathrm{kg}$ Siro and 0.3 $\mathrm{mg} / \mathrm{kg}$ Nin, i.p.), starting at the time of tamoxifen injection, significantly reduced GI bleeding (Figure 8, A-D) and partially, but significantly, increased hematocrit level, RBC number, and hemoglobin level (Figure 8, E-G) compared with the vehicle-treated Alk1-iKO controls.

In tBMP9/10ib mice, Siro prevents mTOR overactivation, and Nin blocks overactivated VEGFR2. We next assessed mTOR signaling in tBMP9/10ib mice by measuring the levels of phospho-mTOR (p-mTOR) and phospho-S6 (p-S6) in the liver and retina. Western blot analyses revealed robust increases in p-mTOR and p-S6 levels in whole-liver homogenates isolated from tBMP9/10ib mice compared with control mice (Figure 9A). Treatment with Siro (alone or in combination with Nin) blocked S6 phosphorylation and normalized p-mTOR levels in the tBMP9/10ib mouse liver (Figure 9A and Supplemental Figure 9). Examination of the tBMP9/10ib retinal tissue confirmed the presence of strong p-S6 immunoreactivity in the AVMs, which could significantly be blocked by Siro treatment of the mice (Figure 9, B and C).

A significant elevation of activated phospho-VEGFR2 (p-VEGFR2) was detected in protein homogenates of liver ECs isolated from tBMP9/10ib mice (Figure 9D and Supplemental Figure 10) and in the tBMP9/10ib mouse retina (Figure 9E), which was significantly inhibited by Nin treatment of the mice (Figure 9, D-F, and Supplemental Figure 11). We also verified that the drug combination did not interfere with the inhibitory effect of Nin on p-VEGFR2. As observed upon treatment with Nin alone, Siro + Nin fully inhibited VEGF-induced VEGFR2 activation in primary ECs (Supplemental Figure 2). Altogether, these data confirmed that endothelial mTOR and VEGFR2 were overactivated in the tBMP9/10ib mice and that Siro + Nin could respectively and efficiently block these signaling deregulations in vivo.

Siro rescues Smad1/5/8 signaling by activating ALK2. Because the possibility that Siro might both prevent mTOR overactivation and rescue Smad1/5/8 signaling in HHT mice is appealing, we revisited the effect of Siro on Smad1/5/8 signaling in cell culture systems. We found that Siro increased Smad1/5/8 phosphorylation (p-Smad1/5/8) in a dose-dependent manner and increased the levels of the Smad1/5/8 downstream target ID1 (inhibitor of differentiation 1) in C2C12 myoblast cells (Supplemental Figure 12A) and in human umbilical vein ECs (HUVECs, Figure 10A), at doses very comparable to Tac (32). A pharmacological approach using the pan-ALK inhibitor LDN-193189 (Figure 10B) and gene silencing (Figure 10C and Supplemental Figure 12B) in HUVECs further showed that ALK2 is required for the stimulatory effect of Siro on Smad1/5/8 signaling.

To further demonstrate efficacy in a cell culture system more directly relevant to the pathophysiology of human HHT, we used 


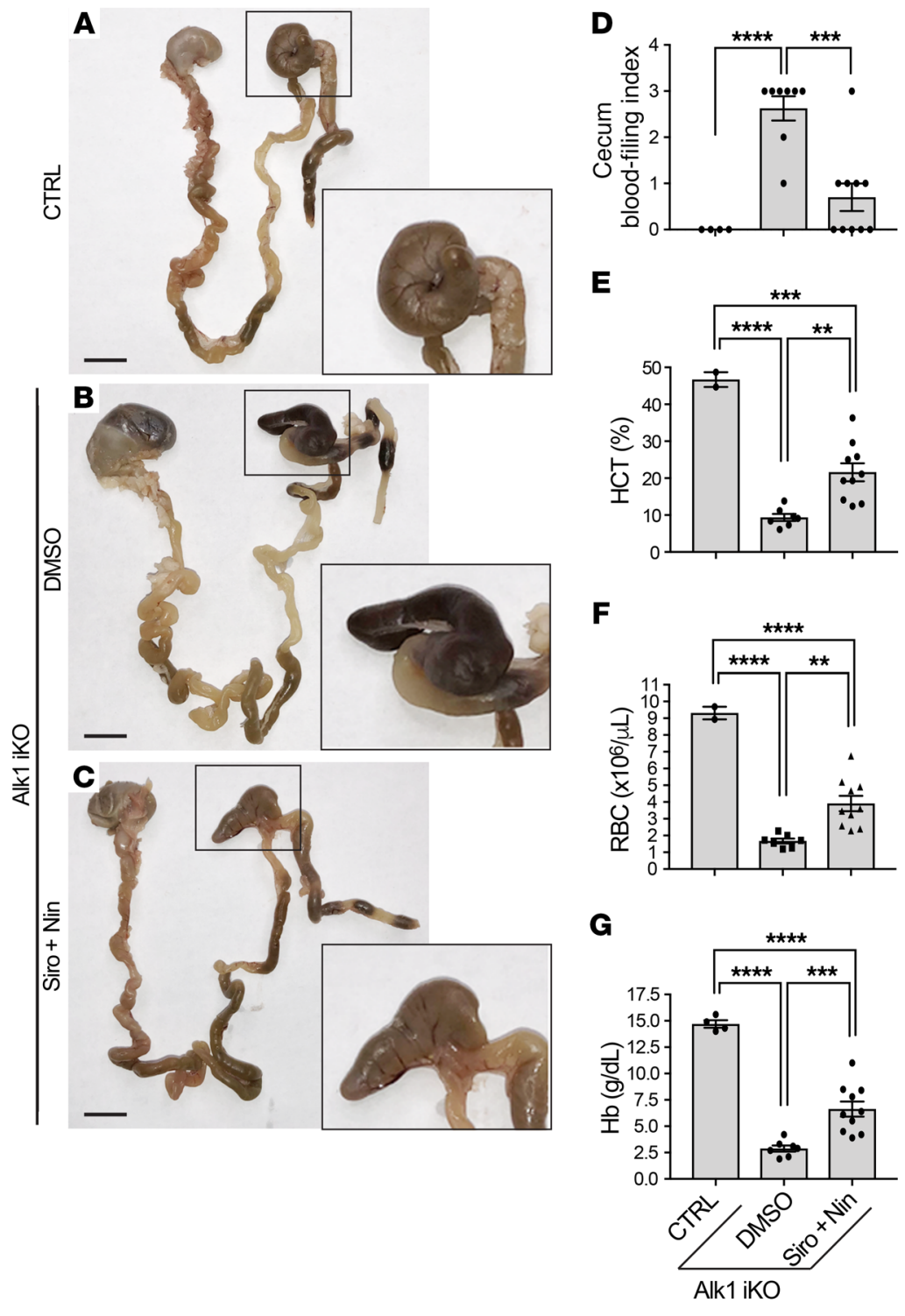

Figure 8. Siro + Nin effectively reduces GI bleeding and ameliorates anemia in adult Alk1-iKO mice. (A-C) GI tract from stomach to rectum of 2- to 4-month-old CreER-negative control (CTRL; A) and Alk1-iKO mice treated with vehicle (DMSO; B) or Siro + Nin (C). Higher magnifications show the cecum. Scale bars: $10 \mathrm{~mm}$ (A-C). (D-C) Scatter plots measuring cecum bleeding index (D), hematocrit (HCT) level (E), red blood cell (RBC) number $(\mathbf{F})$, and hemoglobin $(\mathrm{Hb})$ level $(\mathbf{C})$. Data represent mean \pm SEM $(n=2-4,7-8$, and 10 mice for the CTRL, DMSO, and Siro + Nin groups, respectively); 1-way ANOVA (D-F) and 2-way ANOVA (C), Tukey's multiple-comparisons test. ${ }^{* *} P<0.01$; ${ }^{* *} P<0.001 ;{ }^{* * *} P<0.0001$. CBC parameters of the controls were within the expected ranges for adult mice.

blood outgrowth ECs (BOECs) isolated from a patient with HHT2 genetically confirmed to carry the disease-causing ALK1 T372fsX truncation (32). Treating these HHT2 patient BOECs with Siro also increased p-Smad1/5/8 and ID1 levels (Figure 10D). Finally, IHC analyses showed that in vivo Siro was able to modestly but significantly rescue the decrease in $\mathrm{p}-\mathrm{Smad} 1 / 5 / 8$ in the retinal vasculature (Figure 11, A and B, and Supplemental Figure 13). Thus, Siro displayed potent in vitro Smad1/5/8 signaling activating properties in EC cultures, including in HHT patient-derived ECs, and significantly rescued Smad1/5/8 loss of function in vivo in tBMP9/10ib mice.

\section{Discussion}

Major progress in techniques of interventional radiology, embolization, and cauterization have considerably reduced the risk of hemorrhage in patients with HHT. However, the recurring and progressive nature of the disease continues to make HHT a highly debilitating disorder associated with life-threatening sequelae. Several disease-modifying therapies have been proposed in preclinical models, and some of them are currently being investigated in clinical trials (49). Notably, Tac was found to be associated with Smad1/5/8-activating properties in ECs and showed some efficacy in reducing pathology in HHT (32) and PAH (37) models and patients (50-52). Here we propose that Siro, a structural analog of Tac and inhibitor of mTOR, is a more potent blocker of AVM development in HHT mice. We found that Siro acts as a dual modulator by stimulating Smad1/5/8 while inhibiting mTOR, in cell cultures and HHT mice, thus correcting 2 key signaling defects of the HHT ECs.

In cell cultures, Tac was proposed to activate Smad1/5 by facilitating dissociation of the binding repressor FKBP12 from ALK1, ALK2, and ALK3, while Siro only recruited ALK2 (37). Here, we confirmed in HUVECs that Siro could activate endothelial Smad1/5/8 by specifically stimulating ALK2. Although these data would need to be confirmed in vivo, they suggest the intriguing possibility that ALK2 activation might compensate for ALK1 loss of function. Although attractive from a mechanistic aspect, this mechanism might be risky because ALK2activating mutations are associated with fibrodysplasia ossificans progressiva, a disorder characterized by progressive heterotopic bone formation in muscle tissues (53). However, both Tac and Siro are not known to lead to any adverse effects associated with mechanisms of abnormal ossification in humans. In addition, Siro showed a potent anti-AVM effect at doses (9-22 nM in mouse serum) comparable to its use in the clinical setting (whole-blood trough concentrations of $\sim 15-20 \mathrm{nM}$ for prophylaxis of transplant rejection, for instance).

Our data highlight a beneficial interaction and synergy between Siro and Nin treatments in HHT mice. This interaction was observed by RNA sequencing (RNA-Seq) of the tBMP9/10ibliver, which revealed the presence of deregulated genes that were fully normalized only when Siro and Nin were coadministered. This transcriptional interaction translated into a robust synergy of the 2 drug treatments and a significant improvement of the Siro effect at preventing AVMs in HHT mice. We found that Nin acted, at least in part, by blocking the overactivation of VEGFR2 in ECs of the tBMP9/10ib mice. Nin is not specific to VEGFRs, as it also targets FGFRs and PDGFRs, 2 other receptor families involved in angiogenesis (54). Further investigation will be needed to determine whether interference of these other RTK receptor pathways might be involved in the antiAVM effect of the Siro + Nin combination. Previous work suggests, 
A

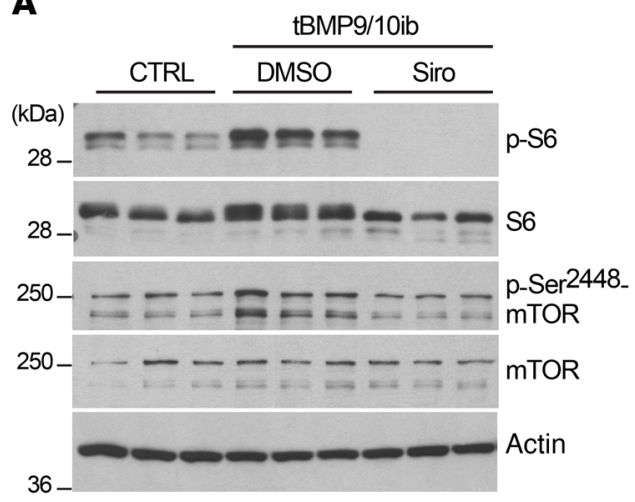

B

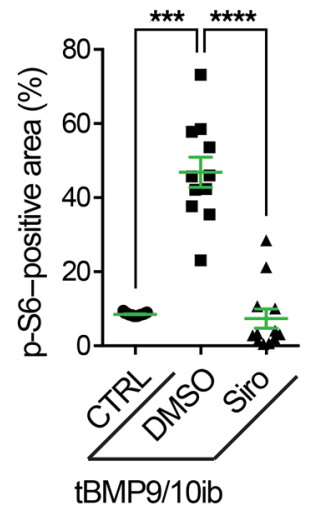

C
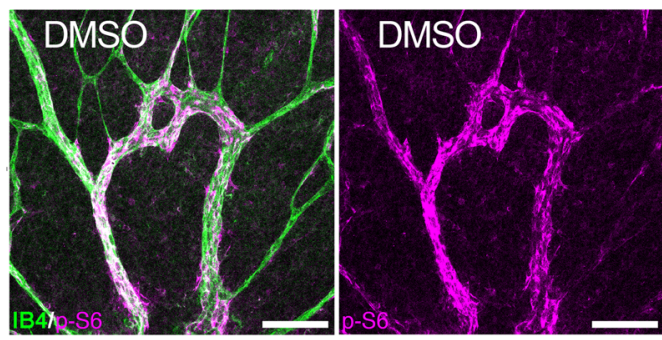

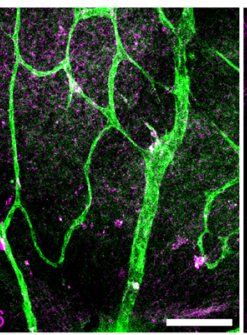

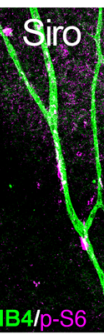

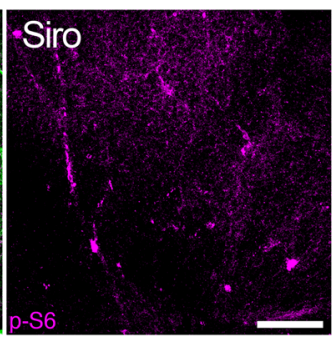

D

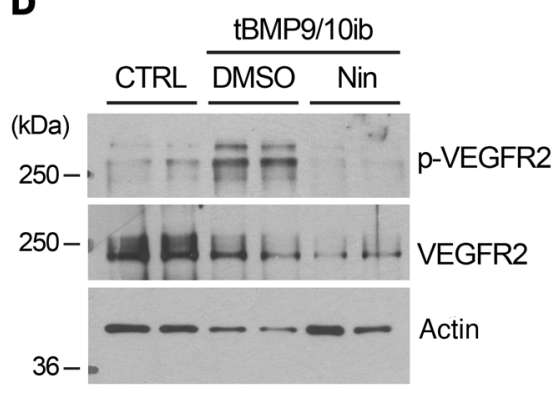

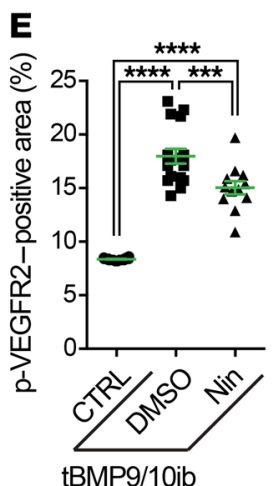

tBMP9/10ib
Figure 9. Siro prevents mTOR overactivation, and Nin blocks overactivated VEGFR2 in tBMP9/10ib mice. (A-F) Control (CTRL) and tBMPg/10ib mice were treated with DMSO and Siro $(\mathbf{A}-\mathbf{C})$ or Nin $(\mathbf{D}-\mathbf{F})$. Protein homogenates of whole liver from $\mathrm{P} 6$ mice $(\mathbf{A})$ and of liver ECs isolated from P9 mice (D) were analyzed by Western blot using antibodies directed against the indicated proteins. Scatter plots show areas positive for $\mathrm{p}-\mathrm{S} 6$ (B) and p-VEGFR2 (E) analyzed by IHC in the retina of P6 CTRL and tBMPg/10ib mice treated with DMSO and Siro (B) or Nin (E), expressed as a percentage of the retinal vascular area occupied by immunofluorescence staining. Data represent mean $\pm \operatorname{SEM}(n=4,3$, and 3 mice for the CTRL, DMSO, and Siro [B] or Nin [E] groups, respectively); p-S6 analysis: Kruskal-Wallis test, post hoc Dunn's multiple-comparisons test; $p$-VEGFR2 analysis: 1-way ANOVA, Tukey's multiple-comparisons test. ${ }^{* *} P$ $<0.001$; ${ }^{* * *} P<0.0001$. Representative immunofluorescence images show $\mathrm{P} 6$ retinas from tBMP9/10ib mice treated as in B and $\mathbf{E}$ stained with fluorescent isolectin B4 (green, C and F), anti-p-S6 (magenta, C), anti-pVEGFR2 (red, F), and anti-Erg (white, F) antibodies. Scale bars: $100 \mu \mathrm{m}(\mathbf{C})$ and $50 \mu \mathrm{m}$ (F).

$\mathbf{F}$
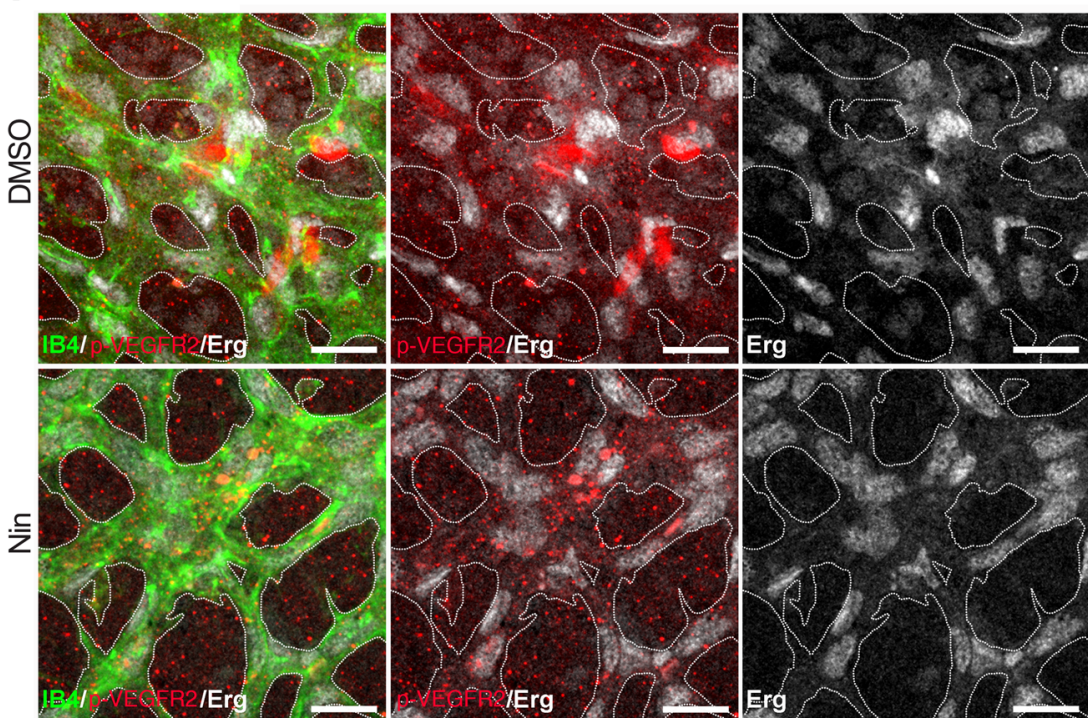

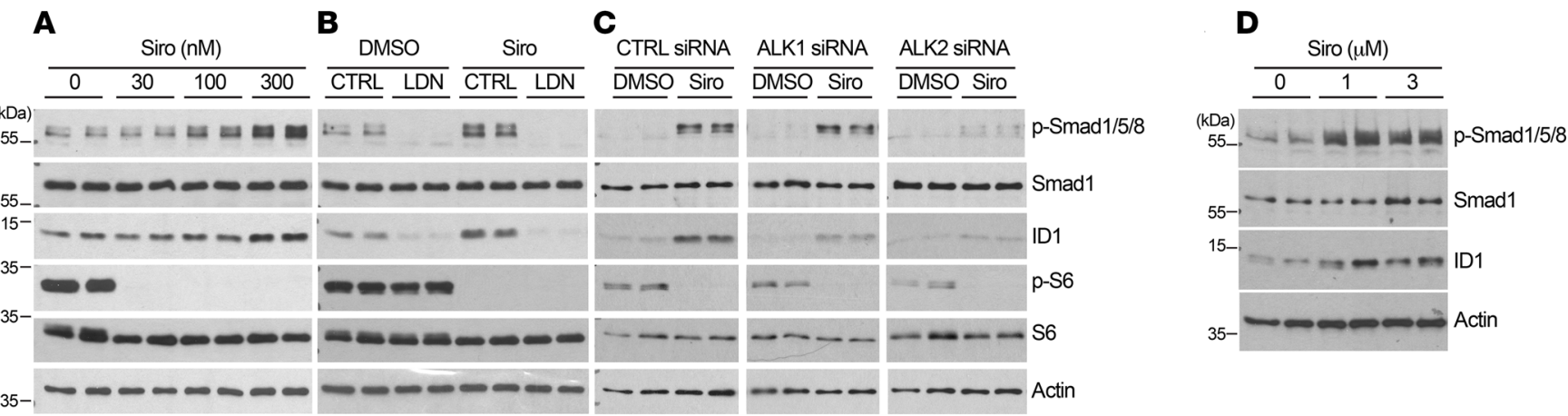

Figure 10. Siro activates Smad1/5/8 via ALK2. (A-D) HUVECs (A-C) and HHT2 patient-derived BOECs (D) were treated for 3 hours with vehicle (DMSO) or Siro in complete medium (conditioned for 2-3 days) and at the indicated concentrations ( $\mathbf{A}$ and $\mathbf{D}$ ) or at $300 \mathrm{nM}$ (B and $\mathbf{C}$ ), in the absence or presence of LDN-193189 $(\mathrm{LDN}, 1 \mu \mathrm{M}, \mathbf{B})$, or the indicated siRNA treatments (C). Cell extracts were analyzed by Western blot using antibodies directed against the indicated proteins.

however, that this might not be the case for PDGFRs, since PDGF signaling activation (not inhibition) was found to mitigate vessel wall defects in an Eng-KO mouse model (55).

The current data provide further evidence that HHT pathogenesis requires the overactivation of the mTOR and VEGFR2 pathways (Figure 11C). How Smad1/5/8, mTOR, and VEGFR2 control each other during the disease process remains unclear. Although loss-of-function mutations in BMP9/ALK1/endoglin/ Smad4 signaling are necessary to cause HHT, they are not sufficient. Indeed, additional genetic, environmental, and/or developmental modifiers are required for AVM development, a process referred to as the double/multiple hit hypothesis (56). Notably, VEGF stimulation or wounding, 2 triggers of angiogenesis, were reported to be required to generate brain and skin AVMs in adult ALK1-deficient mice (43). Furthermore, in HHT neonate mouse models, including in tBMP9/10ib pups, it is believed that retinal AVMs develop spontaneously and aggressively because vessel development is actively occurring through angiogenesis. Synergy between BMP9/10 blocking and VEGFR2 activation during the normal process of angiogenic vascular development would thus provide in these models the ideal milieu for lesion development. Therefore, although there is now strong evidence that Smad1/5/8 reduction overactivates VEGF signaling to cause vascular pathology, these 2 pathways are initiated by independent triggers. Our data showing that Nin and Siro treatments have clear synergistic effects support this notion and further show that the 2 pathways can independently signal in ECs to cause AVMs.

Recent studies have demonstrated that PI3K/Akt signaling is overactivated in several HHT models, and that its inhibition reduces the $\operatorname{AVMs}(25,30,31)$. In line with this observation, we show here that, in the tBMP9/10ib mouse liver, there is an

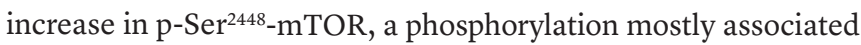
with mTORC1, the mTOR complex downstream from PI3K/ Akt, which is directly targeted by Siro (57). In addition, Siro treatment, which fully prevented p-Ser ${ }^{2448}$-mTOR and S6 overactivation, reduced AVM number in our model, suggesting that mTOR, most likely downstream from PI3K/Akt, is a major player in AVM development. We further found that, although Siro fully inhibited mTOR overactivation in vivo, its anti-AVM effect was only partial and could be significantly potentiated upon VEGFR2 inhibition by Nin, indicating that VEGFR2 can signal independently of the
PI3K/Akt/mTOR pathway to control the AVMs. This observation concurs with recent data obtained in Smad4-deficient mice, which indicate that abnormal PI3K/Akt activation in ECs is primarily controlled by blood flow and not VEGFR2 $(24,58)$.

An important question relates to the drug dosing used in this study and whether it allows the administered drugs to reach concentrations within the expected therapeutic range. We measured an average of $9.0 \mathrm{nM}$ Siro and $5.3 \mathrm{nM}$ Nin at P6, and an increase to 22.6 nM Siro and $24.7 \mathrm{nM}$ Nin at P9 in the pup serum. Previously published in vitro experiments reported a Siro $\mathrm{IC}_{50}$ of approximately $0.1 \mathrm{nM}$ for mTOR (59) and a Nin $\mathrm{IC}_{50}$ of approximately 13 $\mathrm{nM}$ for VEGFR2 (60). At the tested dosing, circulating concentrations of Siro and Nin are therefore expected to inhibit in vivo mTOR and VEGFR2, which is in line with the observed effects on these targets in tBMP9/10ib mice (Figure 9). For Smad1/5/8 stimulation by Siro, our experiments in HUVECs showed that the lowest effective concentration is $100 \mathrm{nM}$ (Figure 10A), which is about 4 times higher than the concentration detected in the serum. We found nonetheless that Siro treatment modestly but significantly increased $\mathrm{p}-\mathrm{Smad} 1 / 5 / 8$ in the mouse retina, indicating that the drug was delivered in sufficient quantities to stimulate Smad1/5/8 signaling in vivo (Figure 11). We have not obtained clear experimental data that explain this apparent discrepancy. However, it is important to note that concentrations used in vitro and drug serum concentrations are difficult to compare. Indeed, drug concentration measurements in the circulation do not consider the pharmacokinetics and bioavailability of the drugs. One important parameter is cellular uptake and accumulation, which can significantly reduce serum concentration while increasing local drug delivery. Altogether, these data suggest that the administered drugs reached concentrations within the expected therapeutic range.

We found that Siro + Nin treatment prevented anemia in P9 tBMP9/10ib pups and significantly improved the CBC parameters in adult Alk1-iKO mice (Figure $2 \mathrm{~B}$ and Figure $8, \mathrm{D}-\mathrm{G}$ ). In tBMP9/10ib pups, drug treatment generated a supranormal elevation of the CBC parameters. In some models of anemia, Siro was reported to ameliorate anemia by increasing stress erythropoiesis (61). In this context, the supranormal elevation of the CBC parameters measured after drug treatment could be due, in part, to changes in erythropoiesis. To test this hypothesis, additional 
A
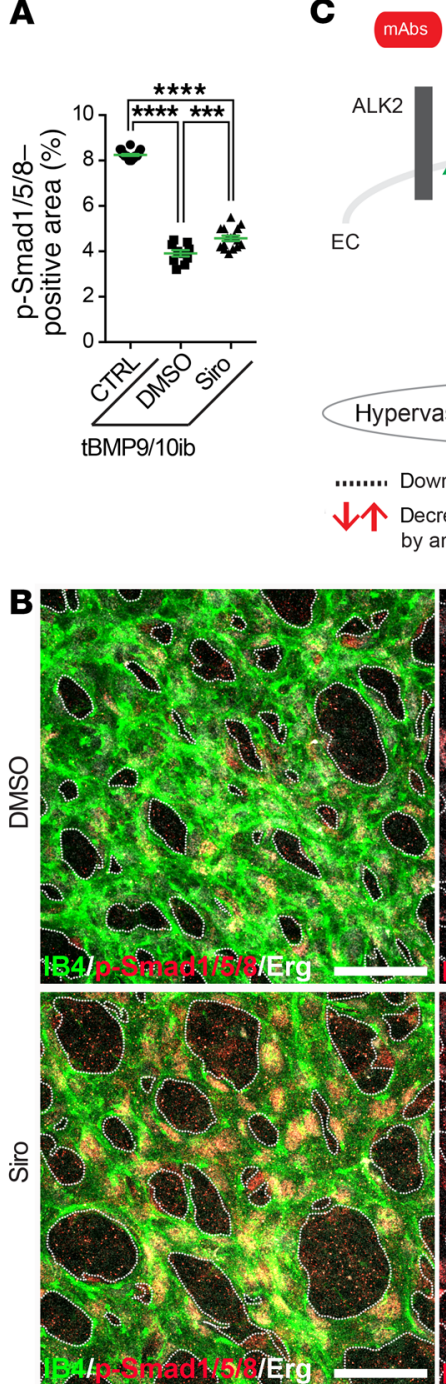
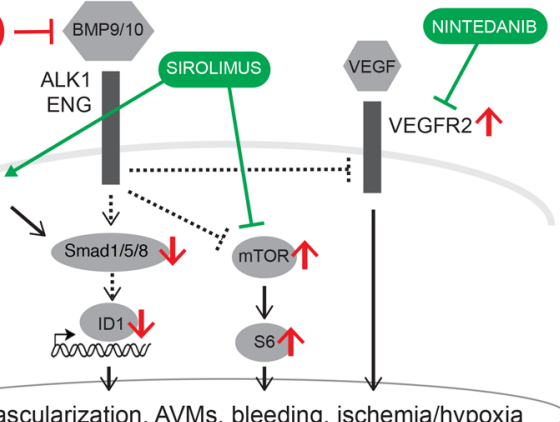
ALK1
ENG

Downregulated by anti-BMP9/10 mAbs Decreased/increased activity/level y anti-BMP9/10 mAbs
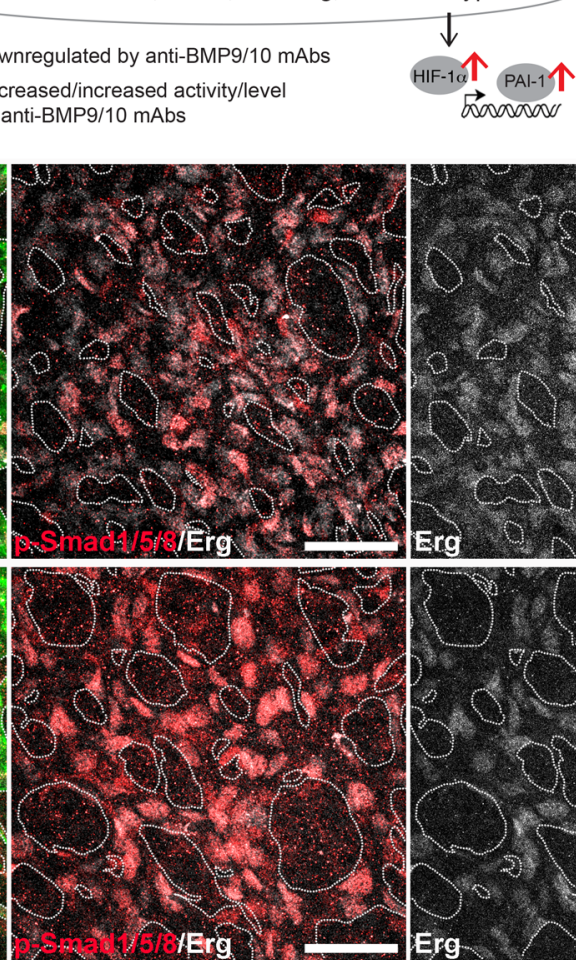
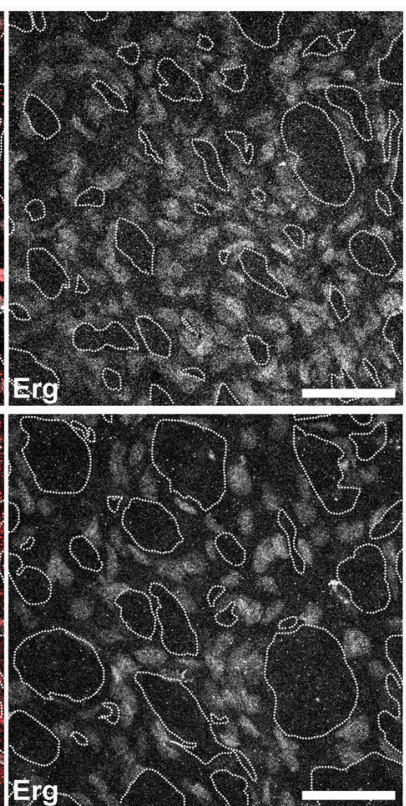

Figure 11. Siro rescues Smad1/5/8 signaling in the tBMPg/10ib retina. (A) Scatter plot measuring $\mathrm{p}-\mathrm{Smad} 1 / 5 / 8-$ positive area in the retina of $\mathrm{P} 6$ control (CTRL) and tBMP9/10ib mice treated with DMSO or Siro, expressed as a percentage of the retinal vascular area occupied by immunostaining. Data represent mean $\pm \operatorname{SEM}(n=4,3$, and 3 mice for the CTRL, DMSO, and Siro groups, respectively). ${ }^{* * *} P<0.001$, ${ }^{* * *} P<0.0001$; 1-way ANOVA, Tukey's multiple-comparisons test. (B) Representative images showing P6 retinas from tBMP9/10ib mice treated as in $\mathbf{A}$ stained with fluorescent isolectin B4 (green), anti-Erg (white), and antip-Smad1/5/8 (red). Scale bars: $50 \mu \mathrm{m}$. (C) Schematic illustration of the proposed synergistic effects of Siro and Nin on endothelial Smad1/5/8, mTOR, and VEGFR2 signaling pathways. experiments were performed by using the recently described method of monitoring erythropoiesis using CD44/Ter119/FSC as markers of terminal erythroid differentiation (62). We measured a significant increase in the number of reticulocytes in the bone marrow, but not in the spleen, of Siro + Nin-treated tBMP9/10ib mice (Supplemental Figure 14). These data suggest that although no stress erythropoiesis was observed, an increase in RBC production occurred and thus could explain, at least partly, the supranormal levels of hematocrit, RBCs, and hemoglobin measured after the drug treatment. Further investigation will be required to clearly define the origin of the observed effects, for instance, by looking at the earlier RBC precursors (62). Other experiments will also be needed to precisely determine how much of the corrected anemia is driven by a bleeding reduction (Figure $3 \mathrm{M}$ ) versus an increased RBC production (Supplemental Figure 14) and whether the drug effect on RBC production could be related to developmental aspects in newborn mice. In support of the latter possibility, a supranormal elevation of the RBC counts was not found in treated adult $A l k 1$-iKO mice (Figure 8), further supporting our current interpretation that anemia correction is primarily driven by a bleeding reduction.
Prophylaxis of rejection in liver transplantation includes treatment with immunosuppressive drugs, primarily Tac and more rarely Siro. Recently, liver AVM pathology recurrence was reported in patients with HHT who underwent liver transplantation while they were treated with Tac (63). Our study suggests the possibility that prioritizing Siro versus Tac (together or not with Nin) in the antirejection regimen might also help in specifically preventing the spread of vascular disease in healthy liver grafts.

More broadly, the beneficial effect of Siro and mTOR inhibition has already been described in models of pathological angiogenesis (64). In addition, Siro treatment has produced promising results in patients affected by vascular anomalies (65). Our observation that Siro and Nin mechanistically synergize to block the pathogenic process of HHT might thus have important ramifications for the treatment of other vascular disorders, such as the vascular anomaly diseases.

In conclusion, the present data show that Siro + Nin combination treatment concurrently normalizes endothelial Smad1/5/8, mTOR, and VEGFR2 pathways to synergistically and efficiently oppose HHT pathogenesis and the associated vascular pathology in neonate and adult mouse models of the disease. We 
thus propose that Siro + Nin combination treatment has therapeutic potential in HHT.

\section{Methods}

Mice. This study was performed using C57BL/6J mice (The Jackson Laboratory). R26-Cre ${ }^{\mathrm{ER} /+}$ Alk1 ${ }^{2 \mathrm{f} / 2 \mathrm{f}}$ mouse generation and Alk1 deletion using tamoxifen were described previously $(39,48)$. Mice were maintained in regular housing conditions and were allowed free access to water and a maintenance diet.

Transmammary-delivered immunoblocking of BMP9 and BMP1O and drug treatments. tBMP9/10ib pups and age-matched control pups (treated with isotype controls) were generated as previously described $(22,32)$. Pups (litter size, $n=7-9$ mice; average mouse weight, $2 \mathrm{~g}$ ) were treated with vehicle (0.1\% DMSO in saline), Siro $(0.5 \mathrm{mg} / \mathrm{kg} \mathrm{BW})$, and $\mathrm{Nin}(0.3 \mathrm{mg} / \mathrm{kg} \mathrm{BW})$ following the different combinations and schedules described in the figures. Isotype control pups were injected with vehicle.

RNA-Seq, readalignments, andgeneexpressiondifferentialexpression analysis. RNA was processed for RNA-Seq at the Genomics Resources Core Facility, Weill Cornell Medical College, on an Illumina HiSeq 4000 instrument. Alignments of reads to the transcriptome were performed with Kallisto version 0.44.0. We aligned reads to the mouse transcriptome downloaded from Ensembl (Mus_musculus.GRCm38.cdna.all.fa.gz). Alignment was run on a MacOS laptop with 16 GB of memory, using the NextflowWorkbench (66) to orchestrate alignment and count aggregation for the analyzed samples. RNA-Seq transcript counts were analyzed with analysis scripts written with the MetaR languages (67). Reads were deposited in the Sequence Read Archive database of NCBI (BioProject accession PRJNA577399). Additional details of the analyses are described in the Supplemental Methods.

Statistics. For multiple-group comparisons, a 1-way ANOVA test was performed (when data fulfilled the assumption of normality for parametric tests, using a D'Agostino and Pearson omnibus test) and a post hoc Tukey's multiple-comparisons test was used. When normality was not achieved, a nonparametric Kruskal-Wallis test and post hoc Dunn's multiple-comparisons test were used. For 2-group comparisons, when normality was not achieved, a Mann-Whitney test was per- formed instead. $P$ values less than 0.05 were considered statistically significant. Analyses were done using GraphPad Prism.

Study approval. Study subject (HHT2 patient carrying the ALK1 $\mathrm{T} 372 \mathrm{fs} \mathrm{X}$ mutation) provided voluntary and written informed consent using a form approved by The Feinstein Institutes for Medical Research Institutional Review Board (IRB). Study subject BOECs were isolated and cultured using a protocol approved by the Institute's IRB. All animal procedures were performed in accordance with protocols approved by The Feinstein Institutes for Medical Research and Barrow Neurological Institute Institutional Animal Care and Use Committees, and conformed to the NIH Guide for the Care and Use of Laboratory Animals and ARRIVE guidelines.

Additional methods are described in the Supplemental Methods.

\section{Author contributions}

PM conceived the study. SR and PM designed and supervised the study. JP and LB performed the hematological studies. HC and SPO performed the experiments in Alk1-iKO mice. LD and PW performed the heart function measurements. PKC and CNM provided the HUVECs. MH and YAA performed the LC-MS. FC performed the bioinformatics analyses. SR, HZ, PC, ANK, RP, and $M G$ performed all the other experiments. SR, LB, FC, and PM wrote the manuscript. CNM and SPO edited the manuscript.

\section{Acknowledgments}

This study was supported by NIH/NHLBI grants R01HL139778 (to PM) and R01HL128525 (to SPO), Department of Defense's Congressionally Directed Medical Research Program (DOD/CDMRP) grant PR161205 (to SPO and PM), a Feinstein Institutes for Medical Research fund (to PM), Leducq foundation (ATTRACT, to SPO), and Barrow Neurological Foundation (to SPO).

Address correspondence to: Philippe Marambaud, PhD, Laboratory of Memory Disorders \& Vascular Biology, Litwin-Zucker Center for Alzheimer's Disease \& Memory Disorders, The Feinstein Institutes for Medical Research, Northwell Health, 350 Community Drive, Manhasset, New York 11030, USA. Phone: 516.562.0425; Email:pmaramba@northwell.edu.
1. Faughnan ME, et al. International guidelines for the diagnosis and management of hereditary haemorrhagic telangiectasia. JMed Genet. 2011;48(2):73-87.

2. Shovlin CL. Hereditary haemorrhagic telangiectasia: pathophysiology, diagnosis and treatment. Blood Rev. 2010;24(6):203-219.

3. Dupuis-Girod S, Bailly S, Plauchu H. Hereditary hemorrhagic telangiectasia: from molecular biology to patient care. J Thromb Haemost. 2010;8(7):1447-1456.

4. McAllister KA, et al. Endoglin, a TGF- $\beta$ binding protein of endothelial cells, is the gene for hereditary haemorrhagic telangiectasia type 1 . Nat Genet. 1994;8(4):345-351.

5. Johnson DW, et al. Mutations in the activin receptor-like kinase 1 gene in hereditary haemorrhagic telangiectasia type 2. Nat Genet. 1996;13(2):189-195.

6. Gallione CJ, et al. A combined syndrome of juvenile polyposis and hereditary haemorrhagic tel- angiectasia associated with mutations in MADH4 (SMAD4). Lancet. 2004;363(9412):852-859.

7. Wooderchak-Donahue WL, et al. BMP9 mutations cause a vascular-anomaly syndrome with phenotypic overlap with hereditary hemorrhagic telangiectasia. Am J Hum Genet. 2013;93(3):530-537.

8. Cai J, Pardali E, Sánchez-Duffhues G, ten Dijke P. BMP signaling in vascular diseases. FEBS Lett. 2012;586(14):1993-2002.

9. Goumans MJ, Zwijsen A, Ten Dijke P, Bailly S. Bone morphogenetic proteins in vascular homeostasis and disease. Cold Spring Harb Perspect Biol. 2018;10(2):a031989.

10. Seki T, Yun J, Oh SP. Arterial endotheliumspecific activin receptor-like kinase 1 expression suggests its role in arterialization and vascular remodeling. Circ Res. 2003;93(7):682-689.

11. Saito T, et al. Structural basis of the human endoglin-BMP9 interaction: insights into BMP signaling and HHT1. Cell Rep. 2017;19(9):1917-1928.

12. Newman JH, et al. Genetic basis of pulmonary arterial hypertension: current understanding and future directions. J Am Coll Cardiol. 2004;43(12 suppl S):33S-39S.

13. Massagué J. TGF $\beta$ signalling in context. Nat Rev Mol Cell Biol. 2012;13(10):616-630.

14. Ricard N, et al. Functional analysis of the BMP9 response of ALK1 mutants from HHT2 patients: a diagnostic tool for novel ACVRL1 mutations. Blood. 2010;116(9):1604-1612.

15. Alaa El Din F, et al. Functional and splicing defect analysis of 23 ACVRL1 mutations in a cohort of patients affected by Hereditary Hemorrhagic Telangiectasia. PLoS One. 2015;10(7):e0132111.

16. Mallet C, et al. Functional analysis of endoglin mutations from hereditary hemorrhagic telangiectasia type 1 patients reveals different mechanisms for endoglin loss of function. Hum Mol Genet. 2015;24(4):1142-1154.

17. Park SO, et al. Real-time imaging of de novo arteriovenous malformation in a mouse model of hereditary hemorrhagic telangiectasia. J Clin 
Invest. 2009;119(11):3487-3496.

18. Mahmoud M, et al. Pathogenesis of arteriovenous malformations in the absence of endoglin. Circ Res. 2010;106(8):1425-1433.

19. Corti $P$, et al. Interaction between alk1 and blood flow in the development of arteriovenous malformations. Development. 2011;138(8):1573-1582.

20. Garrido-Martin EM, et al. Common and distinctive pathogenetic features of arteriovenous malformations in hereditary hemorrhagic telangiectasia 1 and hereditary hemorrhagic telangiectasia 2 animal models - brief report. Arterioscler Thromb Vasc Biol. 2014;34(10):2232-2236.

21. Tual-Chalot S, et al. Endothelial depletion of Acvrl1 in mice leads to arteriovenous malformations associated with reduced endoglin expression. PLoS One. 2014;9(6):e98646.

22. Ruiz S, et al. A mouse model of hereditary hemorrhagic telangiectasia generated by transmammary-delivered immunoblocking of BMP9 and BMP10. Sci Rep. 2016;5:37366.

23. Crist AM, et al. Angiopoietin-2 inhibition rescues arteriovenous malformation in a Smad 4 hereditary hemorrhagic telangiectasia mouse model. Circulation. 2019;139(17):2049-2063.

24. Kim YH, Choe SW, Chae MY, Hong S, Oh SP. SMAD4 deficiency leads to development of arteriovenous malformations in neonatal and adult mice. J Am Heart Assoc. 2018;7(21):e009514.

25. Ola R, et al. SMAD4 prevents flow induced arteriovenous malformations by inhibiting casein kinase 2. Circulation. 2018;138(21):2379-2394.

26. Tual-Chalot S, Oh SP, Arthur HM. Mouse models of hereditary hemorrhagic telangiectasia: recent advances and future challenges. Front Genet. 2015;6:25.

27. Ardelean DS, Letarte M. Anti-angiogenic therapeutic strategies in hereditary hemorrhagic telangiectasia. Front Genet. 2015;6:35.

28. Choi EJ, et al. Enhanced responses to angiogenic cues underlie the pathogenesis of hereditary hemorrhagic telangiectasia 2. PLoS One. 2013;8(5):e63138.

29. Thalgott JH, et al. Decreased expression of vascular endothelial growth factor receptor 1 contributes to the pathogenesis of hereditary hemorrhagic telangiectasia type 2. Circulation. 2018;138(23):2698-2712.

30. Ola R, et al. PI3 kinase inhibition improves vascular malformations in mouse models of hereditary haemorrhagic telangiectasia. Nat Commun. 2016;7:13650.

31. Jin Y, et al. Endoglin prevents vascular malformation by regulating flow-induced cell migration and specification through VEGFR2 signalling. Nat Cell Biol. 2017;19(6):639-652.

32. Ruiz $\mathrm{S}$, et al. Tacrolimus rescues the signaling and gene expression signature of endothelial ALK1 lossof-function and improves HHT vascular pathology. Hum Mol Genet. 2017;26(24):4786-4798.

33. Kim JH, Peacock MR, George SC, Hughes CC. BMP9 induces EphrinB2 expression in endothelial cells through an Alk1-BMPRII/ActRII-ID1/ ID3-dependent pathway: implications for hereditary hemorrhagic telangiectasia type II. Angiogenesis. 2012;15(3):497-509.

34. Guilhem A, et al. Intra-venous bevacizumab in hereditary hemorrhagic telangiectasia (HHT):
A retrospective study of 46 patients. PLoS One. 2017;12(11):e0188943.

35. Iyer VN, et al. Intravenous bevacizumab for refractory hereditary hemorrhagic telangiectasiarelated epistaxis and gastrointestinal bleeding. Mayo Clin Proc. 2018;93(2):155-166.

36. Alsina-Sanchís E, et al. ALK1 loss results in vascular hyperplasia in mice and humans through PI3K activation. Arterioscler Thromb Vasc Biol. 2018;38(5):1216-1229.

37. Spiekerkoetter E, et al. FK506 activates BMPR2, rescues endothelial dysfunction, and reverses pulmonary hypertension. J Clin Invest. 2013;123(8):3600-3613.

38. Skaro AI, Marotta PJ, McAlister VC. Regression of cutaneous and gastrointestinal telangiectasia with sirolimus and aspirin in a patient with hereditary hemorrhagic telangiectasia. Ann Intern Med. 2006;144(3):226-227.

39. Kim YH, Kim MJ, Choe SW, Sprecher D, Lee YJ, P Oh S. Selective effects of oral antiangiogenic tyrosine kinase inhibitors on an animal model of hereditary hemorrhagic telangiectasia. J Thromb Haemost. 2017;15(6):1095-1102.

40. Faughnan ME, et al. Pazopanib may reduce bleeding in hereditary hemorrhagic telangiectasia. Angiogenesis. 2019;22(1):145-155.

41. Kovacs-Sipos E, Holzmann D, Scherer T, Soyka MB. Nintedanib as a novel treatment option in hereditary haemorrhagic telangiectasia. BMJ Case Rep. 2017;2017:bcr-2017-219393.

42. Laplante M, Sabatini DM. mTOR signaling in growth control and disease. Cell. 2012;149(2):274-293.

43. Roman BL, Hinck AP. ALK1 signaling in development and disease: new paradigms. Cell Mol Life Sci. 2017;74(24):4539-4560

44. Letteboer TG, et al. Genotype-phenotype relationship in hereditary haemorrhagic telangiectasia. JMed Genet. 2006;43(4):371-377.

45. Kietzmann T, Roth U, Jungermann K. Induction of the plasminogen activator inhibitor-1 gene expression by mild hypoxia via a hypoxia response element binding the hypoxia-inducible factor-1 in rat hepatocytes. Blood. 1999;94(12):4177-4185.

46. Semenza GL. HIF-1: mediator of physiological and pathophysiological responses to hypoxia. JAppl Physiol. 2000;88(4):1474-1480.

47. Warde-Farley D, et al. The GeneMANIA prediction server: biological network integration for gene prioritization and predicting gene function. Nucleic Acids Res. 2010;38(Web Server issue):W214-W220.

48. Park SO, et al. ALK5- and TGFBR2-independent role of ALK1 in the pathogenesis of hereditary hemorrhagic telangiectasia type 2. Blood. 2008;111(2):633-642.

49. Vorselaars VMM, et al. Pulmonary arterial hypertension and hereditary haemorrhagic telangiectasia. Int J Mol Sci. 2018;19(10):E3203.

50. Albiñana V, Sanz-Rodríguez F, Recio-Poveda L, Bernabéu C, Botella LM. Immunosuppressor FK506 increases endoglin and activin receptorlike kinase 1 expression and modulates transforming growth factor- $\beta 1$ signaling in endothelial cells. Mol Pharmacol. 2011;79(5):833-843.

51. Spiekerkoetter E, et al. Low-Dose FK506 (Tacro- limus) in end-stage pulmonary arterial hypertension. Am J Respir Crit Care Med. 2015;192(2):254-257.

52. Sommer N, et al. Treatment with low-dose tacrolimus inhibits bleeding complications in a patient with hereditary hemorrhagic telangiectasia and pulmonary arterial hypertension. Pulm Circ. 2019;9(2):2045894018805406.

53. Fukuda T, et al. Constitutively activated ALK2 and increased SMAD1/ 5 cooperatively induce bone morphogenetic protein signaling in fibrodysplasia ossificans progressiva. J Biol Chem. 2009;284(11):7149-7156.

54. Carmeliet P, Jain RK. Molecular mechanisms and clinical applications of angiogenesis. Nature. 2011;473(7347):298-307.

55. Lebrin F, et al. Thalidomide stimulates vessel maturation and reduces epistaxis in individuals with hereditary hemorrhagic telangiectasia. Nat Med. 2010;16(4):420-428.

56. Ruiz-Llorente L, Gallardo-Vara E, Rossi E, Smadja DM, Botella LM, Bernabeu C. Endoglin and alk1 as therapeutic targets for hereditary hemorrhagic telangiectasia. Expert Opin Ther Targets. 2017;21(10):933-947.

57. Saxton RA, Sabatini DM. mTOR signaling in growth, metabolism, and disease. Cell. 2017;169(2):361-371.

58. Crist AM, Lee AR, Patel NR, Westhoff DE, Meadows SM. Vascular deficiency of Smad4 causes arteriovenous malformations: a mouse model of hereditary hemorrhagic telangiectasia. Angiogenesis. 2018;21(2):363-380.

59. Edwards SR, Wandless TJ. The rapamycin-binding domain of the protein kinase mammalian target of rapamycin is a destabilizing domain. JBiol Chem. 2007;282(18):13395-13401.

60. Hilberg F, et al. BIBF 1120: triple angiokinase inhibitor with sustained receptor blockade and good antitumor efficacy. Cancer Res. 2008;68(12):4774-4782.

61. Beneduce $\mathrm{E}$, et al. Fyn kinase is a novel modulator of erythropoietin signaling and stress erythropoiesis. Am J Hematol. 2019;94(1):10-20.

62. Chen K, Liu J, Heck S, Chasis JA, An X, Mohandas $\mathrm{N}$. Resolving the distinct stages in erythroid differentiation based on dynamic changes in membrane protein expression during erythropoiesis. Proc Natl Acad Sci US A. 2009;106(41):17413-17418.

63. Dumortier J, et al. Recurrence of hereditary hemorrhagic telangiectasia after liver transplantation: clinical implications and physiopathological insights. Hepatology. 2019;69(5):2232-2240.

64. Karar J, Maity A. PI3K/AKT/mTOR pathway in angiogenesis. Front Mol Neurosci. 2011;4:51.

65. Adams DM, et al. Efficacy and safety of sirolimus in the treatment of complicated vascular anomalies. Pediatrics. 2016;137(2):e20153257.

66. Kurs JP, Simi M, Campagne F. NextflowWorkbench: Reproducible and reusable workflows for beginners and experts. doi: https://doi. org/10.1101/041236. Posted on bioRxiv March $28,2016$.

67. Campagne F, Digan WER, Simi M. MetaR: simple, high-level languages for data analysis with the R ecosystem [in press]. doi: https://doi. org/10.1101/030254. Posted on bioRxiv November 4,2015 . 